\title{
Interstellar gas, dust and diffuse bands in the SMC $\mathrm{S}^{\star \star \star}$
}

\author{
N. L. J. Cox ${ }^{1,5}$, M. A. Cordiner ${ }^{2,3}$, P. Ehrenfreund ${ }^{4}$, L. Kaper ${ }^{5}$, P. J. Sarre ${ }^{3}$, B. H. Foing ${ }^{6}$, M. Spaans ${ }^{7}$, \\ J. Cami ${ }^{8}$, U. J. Sofia ${ }^{9}$, G. C. Clayton ${ }^{10}$, K. D. Gordon ${ }^{11}$, and F. Salama ${ }^{12}$ \\ 1 Herschel Science Centre, European Space Astronomy Centre, Research and Scientific Support Department of ESA, \\ Villafranca del Castillo, Ap. de Correos 50727, 28080 Madrid, Spain \\ e-mail: Nick.Cox@esa.int \\ 2 Astrophysics Research Centre, School of Mathematics and Physics, Queen's University Belfast, Belfast, BT7 1NN, \\ Northern Ireland \\ 3 School of Chemistry, The University of Nottingham, University Park, Nottingham, NG7 2RD, UK \\ 4 Astrobiology Group, Leiden Institute of Chemistry, Leiden University, Einsteinweg 55, 2300 RA Leiden, The Netherlands \\ 5 Astronomical Institute, University of Amsterdam, Kruislaan 403, 1098 SJ Amsterdam, The Netherlands \\ 6 ESA/SCI-SR, ESTEC, PO Box 299, 2200 AG Noordwijk, The Netherlands \\ 7 Kapteyn Astronomical Institute, University of Groningen, PO Box 800, 9700 AV Groningen, The Netherlands \\ SETI Institute, 515 North Whisman Road, Mountain View, CA 94043, USA \\ 9 Astronomy Department, Whitman College, Walla Walla, WA 99362, USA \\ 10 Dept. of Physics \& Astronomy, Louisiana State University, Baton Rouge, LA 7080, USA \\ 11 Steward Observatory, University of Arizona, Tucson, AZ 85721, USA \\ 12 Space Science Division, NASA Ames Research Center, Mail Stop 245-6, Moffett Field, California 94035, USA
}

Received 23 March 2006 / Accepted 19 April 2007

\section{ABSTRACT}

\begin{abstract}
Aims. In order to gain new insight into the unidentified identity of the diffuse interstellar band (DIB) carriers, this paper describes research into possible links between the shape of the interstellar extinction curve (including the $2175 \AA$ bump and far-UV rise), the presence or absence of DIBs, and physical and chemical conditions of the diffuse interstellar medium (gas and dust) in the Small Magellanic Cloud (SMC).

Methods. We searched for DIB absorption features in VLT/UVES spectra of early-type stars in the SMC whose reddened lines-ofsight probe the diffuse interstellar medium of the SMC. Apparent column density profiles of interstellar atomic species (Na I, K I, Ca II and $\mathrm{Ti}$ II) are constructed to provide information on the distribution and conditions of the interstellar gas.

Results. The characteristics of eight DIBs detected toward the SMC wing target AzV 456 are studied and upper limits are derived for the DIB equivalent widths toward the SMC stars AzV 398, AzV 214, AzV 18, AzV 65 and Sk 191. The amount of reddening is derived for these SMC sightlines, and, using $R_{\mathrm{V}}$ and the $\mathrm{H}$ I column density, converted into a gas-to-dust ratio. From the atomic column density ratios we infer an indication of the strength of the interstellar radiation field, the titanium depletion level and a relative measure of turbulence/quiescence. The presence or absence of DIBs appears to be related to the shape of the extinction curve, in particular with respect to the presence or absence of the $2175 \AA$ feature. Our measurements indicate that the DIB characteristics depend on the local physical conditions and chemical composition of the interstellar medium of the SMC, which apparently determine the rate of formation (and/or) destruction of the DIB carriers. The UV radiation field (via photoionisation and photo-destruction) and the metallicity (i.e. carbon abundance) are important factors in determining diffuse band strengths which can differ greatly both between and within galaxies.
\end{abstract}

Key words. astrochemistry - galaxies: Magellanic Clouds - ISM: dust, extinction - ISM: lines and bands - ISM: molecules ISM: atoms

\section{Introduction}

Recent observations of diffuse interstellar bands (DIBs) in Galactic and extra-galactic environments have provided new insight into the conditions that determine their presence in the interstellar media of different types of galaxies. DIBs have been observed in spectra probing the dust-containing diffuse interstellar medium throughout the Galaxy and have been reported

\footnotetext{
* Based on observations collected with VLT/UVES at the European Southern Observatory, Paranal, Chile (ESO programs 67.C-0281 and 71.C-0679).

$\star \star$ Tables 7-9 and Fig. 8 are only available in electronic form at http://www. aanda.org
}

in extragalactic systems as well (e.g. Rich 1987; Vladilo et al. 1987; Morgan 1987; D’Odorico et al. 1989; Heckman \& Lehnert 2000; Junkkarinen et al. 2004; Sollerman et al. 2005; Cox et al. 2006; Welty et al. 2006; York et al. 2006). The SMC, an irregular (disrupted) dwarf galaxy, offers a unique laboratory where the conditions of the interstellar medium are profoundly different from those in the Galaxy as well as markedly from the Large Magellanic Cloud. For example, it has an average metallicity one tenth that of the Milky Way (Dufour 1984), and the average gas-to-dust ratio is about 10 times larger (e.g. Fitzpatrick 1985; Gordon et al. 2003; Cartledge et al. 2005). These differences have a direct influence on the chemistry and physical conditions of the interstellar gas and dust (e.g. Sofia et al. 2006; 

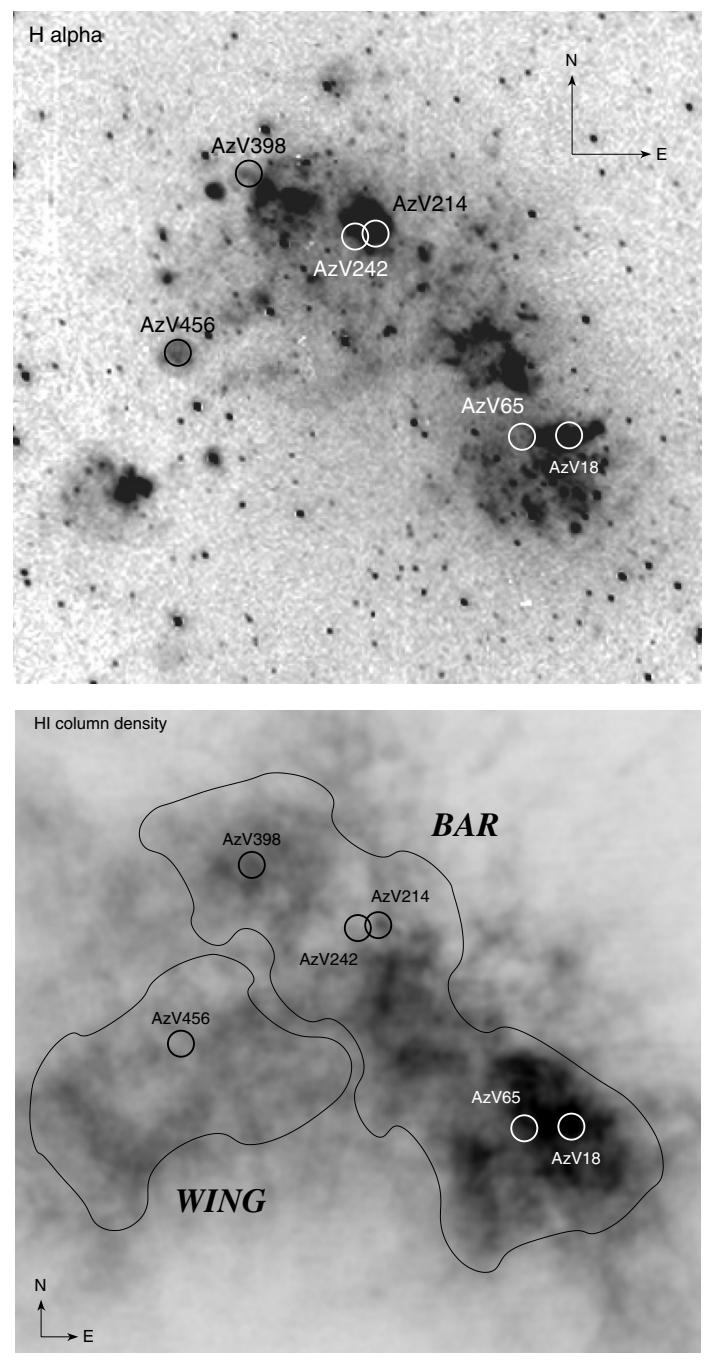

Fig. 1. The observed targets (Table 1) are plotted as black and white circles on two maps of the SMC at different wavelengths. The top map shows $\mathrm{H} \alpha$ (Bothun 1994). The bottom panel shows the map of the H I column density (Stanimirovic et al. 1999, 2000). For $N(\mathrm{H} \mathrm{I})$ the scale is from 0 to $1.03 \times 10^{22}$ atoms $\mathrm{cm}^{-2}$. The maps are aligned in the world coordinate system (North is up, East is to the right), and have the same size $\left(\sim 3^{\circ} \times 3^{\circ}\right)$. Sk 191 is situated outside the lower left corner of the map. The SMC wing and bar region are indicated schematically in the bottom panel.

Cox \& Spaans 2006). Within the SMC different regions can be distinguished such as the SMC bar, an active star forming region, and the SMC wing (Caplan et al. 1996), which is a more quiescent environment associated with modest star formation and less $\mathrm{H} \alpha$ emission. This suggests an environment more protected from the harsh UV field compared to that in the SMC bar (Fig. 1).

The Galactic extinction curve is well described by the extinction law of Cardelli et al. $(1988,1989)$, including the prominent $2175 \AA$ feature. Four out of about 400 Galactic sightlines studied by Valencic et al. $(2003,2004)$ show systematic deviations from this extinction law, and are similar to the average, SMC-like extinction law with a weak or absent $2175 \AA$ feature and a steep farUV rise. Remarkably, these few anomalous Galactic sightlines that sample dense, molecule-rich clouds, produce significantly weaker DIBs than sightlines with similar reddening and standard UV extinction curves (e.g. HD 62542, Ádámkovics et al. 2005; HD 29647, Cardelli \& Savage 1988).
The identity of the DIB carrier(s) remains unknown (see reviews by Herbig 1995; and Sarre 2006), but the current perception is that (electronic) transitions in (possibly charged) organic molecules, e.g. stable aromatic molecules such as PAHs (Van der Zwet \& Allamandola 1985; Leger \& D'Hendecourt 1985; Crawford et al. 1985), fullerenes (Foing \& Ehrenfreund 1994, 1997; Leger et al. 1988) or tubular PAHs (Zhou et al. 2006) are responsible for the several hundred absorption lines (i.e. DIBs) detected in reddened, early-type star spectra. The DIB strength (per unit reddening) seems to depend strongly on the properties of the UV radiation field and possibly also on the shape of the extinction curve. The effect of effective penetration of the UV field is often referred to as the "skin"-effect (Herbig 1995; Cami et al. 1997). The UV-shielded and UV-exposed environments are often termed $\zeta$ and $\sigma$ type clouds, respectively. To further substantiate the relation between DIBs and the (local) environment of the DIB carriers, we have initiated a research program to measure DIB properties in various interstellar environments.

Previous SMC studies have focussed primarily on the 4430 Å DIB, albeit with limited success (Hutchings 1966, 1980; Houziaux et al. 1985; and Nandy et al. 1990). Only recently were the first narrow DIBs unambiguously detected in the SMC wing toward the star AzV 456 (Ehrenfreund et al. 2002), and along several SMC bar sightlines (Welty et al. 2006). The extinction curve in the former SMC sightline is "anomalous" in the sense that it is similar to the average Galactic extinction curve. This observation provides strong motivation for the study of other linesof-sight in the SMC to investigate further the relation between the DIB spectrum and the extinction curve.

In this paper we present high-resolution spectra of targets in the SMC wing and bar obtained with the Very Large Telescope (VLT) and the Ultraviolet-Visual Echelle Spectrograph (UVES) (Sect. 2). We then first describe the near-IR to far-UV extinction curve (Sect. 3). In Sect. 4.1 we present the observed spectra and the search for DIBs in six SMC lines-of-sight. If DIB carriers are indeed carbonaceous molecules in the gas-phase, then it is expected that they coexist with atomic and small molecular species in diffuse interstellar clouds, and are therefore likely to be affected by the same local physical and chemical conditions. The dust and gas properties are considered in Sects. 4.2 and 4.3 based on the extinction and atomic line transitions, respectively. Related properties, such as atomic line ratios and the gas-to-dust ratio are also derived. In Sect. 5 we review the properties of the interstellar gas, dust and diffuse bands in the SMC lines-of-sight and discuss the behaviour (presence/absence) of the DIBs in the context of the local physical conditions of the ISM. In conclusion we present two possible scenarios for the absence/depletion of DIB and $2175 \AA$ A bump carriers in the SMC bar (Sect. 6).

\section{Observations and data reduction}

High-resolution spectra were obtained of seven SMC targets (Fig. 1) with the Ultraviolet and Visual Echelle Spectrograph (UVES) mounted on the Very Large Telescope (VLT) of the European Southern Observatory (ESO), Paranal, Chile. As part of ESO program 67.C-0281 (September 2001) we acquired spectra of 4 bright early type stars in the SMC; AzV 456, AzV 18, Sk 191 and AzV 242. The spectra were obtained with a slit width of $0.6^{\prime \prime}$, and at three different standard UVES settings: $346+580$, $390+564$ and $437+860$, where the numbers indicate the central wavelength (in $\mathrm{nm}$ ) of the blue and red arm, respectively.

A second set of exposures was acquired on three nights in August 2003 (ESO program 71.C-0679). The five targets were 
Table 1. Observational details and target properties. The spectral types, intrinsic colours and reddenings are discussed in Sect. 4.2.

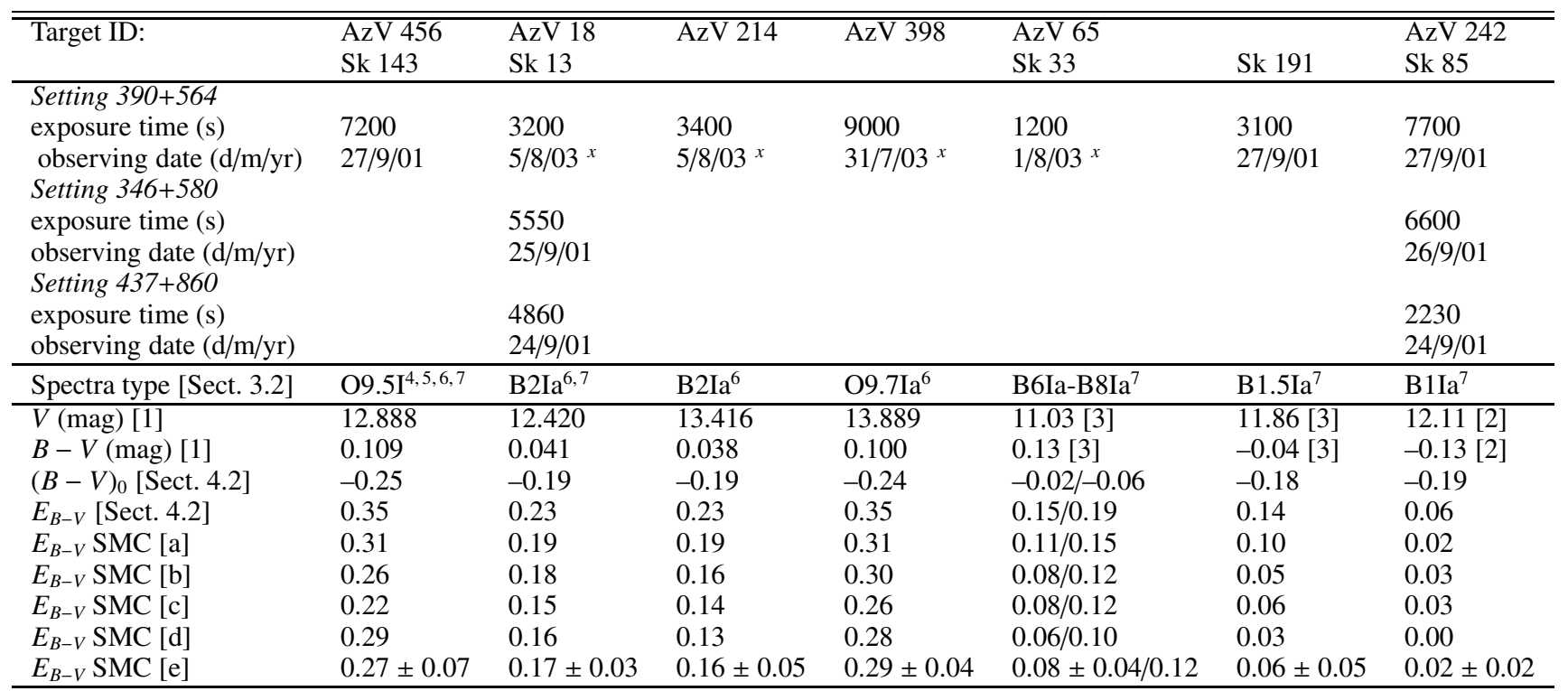

[AzV] catalogue: Azzopardi \& Vigneau (1982); [Sk] catalogue: Sanduleak (1968, 1969). [1] Gordon et al. (2003); [2] Danforth et al. (2002) (D02); [3] Wayte (1990) (W90); [4] Evans (2004; 2dF survey) [5] Evans et al. (2004a); [6] Optical/UV spectral type by Gordon \& Clayton (1998); [7] Lennon (1997); [8] Nandy et al. (1990); [a] MW (Milky Way) foreground reddening is assumed to be 0.04 mag (Sect. 4.2.1). [b] MW and SMC sodium column densities contribute proportionally to the total observed reddening (Sect. 4.3). [c] MW and SMC sodium column densities contribute to the total observed reddening according to $N(\mathrm{NaI})=1.7 \times 10^{14} E_{B-V}^{1.8}$ (Hobbs 1974b), Sect. 4.3. [d] MW foreground reddening is derived via the relation between $N\left(\mathrm{Na}\right.$ I) and $E_{B-V}$ given in [c] and substracted from the total observed reddening (Sect. 4.3). [e] Average $E_{B-V} \mathrm{SMC}$ from methods [a] to [d]. [ $x$ ] UVES with image slicer \#1.

AzV 18, AzV 214, AzV 398, AzV 65 and AzV 242. The spectra were obtained in service mode with image slicer \#1 in the standard UVES setting 390+564. The image slicer chops the observed point source into three slices, each with a width of $0.7^{\prime \prime}$ resulting in a resolving power of about 50000 in the blue arm and 60000 in the red arm. The airmass was about 1.5 for all exposures, and the seeing ranged from $0.66^{\prime \prime}$ to $1.3^{\prime \prime}$ for the different exposures. Observational details and target properties are listed in Table 1.

The data were reduced using the UVES-pipeline (version 2.0.0) within the MIDAS data reduction package (version September 2003). This package provides echelle spectroscopic reduction routines specifically tailored to UVES data. Selected calibration frames (ThAr arc lamp, bias and flatfield exposures) were given as input to the calibration routine (PREP/CALDB) and corresponding output frames and tables were used to reduce the science frames with the optimal extraction routine contained in REDUCE/UVES. The orders were individually extracted. To correct for a residual ripple in the image slicer spectra, which is not removed by the flat field exposure (Dekker et al. 2003), it is necessary to divide the spectrum of the program star by that of the non-reddened comparison star which should preferably be observed at similar conditions (airmass and seeing). Besides (partly) removing the fringe/ripple pattern it simultaneously removes most telluric lines as well. Unfortunately, the signal-tonoise of the resulting spectrum is then reduced by as much as a factor 2 using the available comparison star spectra.

\section{SMC extinction curve: the $2175 \AA$ A UV bump and the far-UV rise}

The extinction curve is a measure of the wavelength dependent attenuation of photons by dust particles, and provides information on the composition and properties of interstellar material along different interstellar sightlines (e.g. Mathis 1990; Draine 2003). One of the most prominent features is the $2175 \AA$ bump; recent studies indicate that it can be attributed to amorphous and hydrogenated amorphous carbon (Mennella et al. 1998; Dartois et al. 2004; Dartois et al. 2005). Fullerenes (Iglesias-Groth 2004), defective carbon onions (Tomita et al. 2004) and PAHs (Duley \& Lazarev 2004) have also been proposed to explain the $2175 \AA$ feature.

For the SMC bar lines-of-sight the interstellar extinction curve is characterised by a very weak $2175 \AA$ bump (2 to 3\% significance for the bump detection, which could also be introduced due to small differences in the Galactic foreground dust between the reddened and the comparison star), and the presence of a strong far-UV non-linear rise (e.g. Rocca-Volmerange et al. 1981; Bromage \& Nandy 1983; Prevot et al. 1984; Gordon \& Clayton 1998; Gordon et al. 2003; and Cartledge et al. 2005). The weakness of the $2175 \AA$ feature in the SMC bar could point toward a harsh environment that triggers the destruction of (carbonaceous) dust into smaller grains or molecules. These would absorb photons at short wavelengths, resulting in increased extinction in the far-UV (Gordon et al. 2003). The SMC-wing lineof-sight AzV 456 does show the $2175 \AA$ bump, and a weak farUV rise, comparable to typical Galactic sightlines. Clayton et al. (2003) fitted dust models to both the SMC bar and SMC wing extinction curve and found that for the latter case their model required a high fraction (even greater than 1) of the available cosmic carbon and silicon. Using additional far-UV extinction observations Cartledge et al. (2005) find the required carbon abundance to be $44 \%$ and $19 \%$ of the available carbon (constrained by the gas-to-dust ratio and the "cosmic" abundance) in order to reproduce the extinction curves toward $\mathrm{AzV} 456$ and $\mathrm{AzV} 18$, respectively. The fraction of amorphous carbon is 


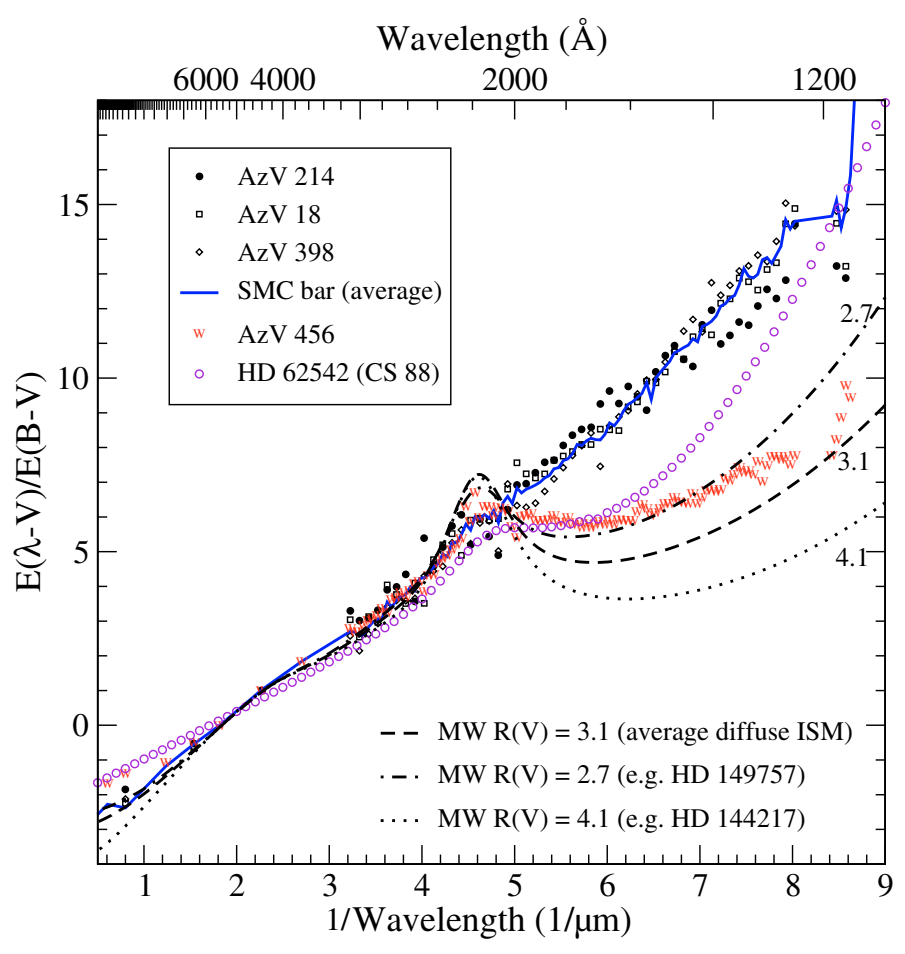

Fig. 2. Extinction curves for the observed SMC targets (adapted from Gordon et al. 2003; Cartledge et al. 2005), and Galactic extinction curves corresponding to $R_{\mathrm{V}}=2.7,3.1$ and 4.1 . Also shown is the Galactic SMC-like extinction curve toward HD 62542.

similar in both, but the graphite fraction is a factor of six smaller in the latter. Both extinction curves require graphite and amorphous carbon as well as astronomical silicates. This indicates an underestimated metal abundance in the line-of-sight toward the SMC wing, which is supported by the lower gas-to-dust ratio. In Fig. 2 we show the extinction curves for the observed reddened targets as published by Gordon et al. (2003; see also earlier work by Rocca-Volmerange et al. 1981; Bromage \& Nandy 1983; Prevot et al. 1984; and Gordon \& Clayton 1998).

Within the Galaxy a few regions show a SMC-like extinction curve (Valencic et al. 2003; Clayton et al. 2000; Sofia et al. 2005). The high far-UV extinction in these clouds may shield the molecules, e.g. fullerenes and PAHs, from both photodissociation (Mathis 1990), and perhaps also photoionisation (Jenniskens et al. 1992). However, these Galactic clouds have higher densities as well as higher extinction compared to those observed in the SMC with their low density, low reddening/extinction, and low metallicity/high gas-to-dust ratios. Cardelli \& Savage (1988) present two of these exceedingly anomalous Galactic interstellar extinction curves in the direction of HD 62542, a line-of-sight associated with a dark cloud in the Gum nebula region, and of HD 29647 in the Taurus dark cloud. The dense region that gives rise to the extinction observed toward HD 62542 is exposed to both intense UV radiation and stellar winds from $\zeta$ Pup and the $\gamma$ Vel system. Strong shocks are undoubtedly also affecting the grains in this region.

HD 62542 shows weak DIBs, for example the 5780 and $5797 \AA$ DIBs are respectively 1.5 and 4 times weaker per unit reddening than for the $\zeta$-type line-of-sight toward HD 149757 (Snow et al. 2002; Ádámkovics et al. 2005; and Table 9). Adamson et al. (1991) show that also HD 29647 displays a weak DIB spectrum for the amount of reddening. They suggest that the relative weakness of these DIBs is driven by changing grain properties rather than by suppression of the UV radiation field.

It is interesting to note that this absence of DIB carriers (absent or very weak DIBs) in both Galactic and SMC sightlines is suggestively linked to the shape of a peculiar extinction curve (e.g. with a weak $2175 \AA$ bump and a steep far-UV rise) found both in the Galaxy and in the SMC. Furthermore, Galactic DIB strengths are detected in the one SMC line-of-sight which shows Galactic-type dust extinction (Ehrenfreund et al. 2002; Welty et al. 2006). We return to this discussion in Sect. 5.

\section{The SMC ISM: DIBs, dust and gas}

\subsection{Diffuse interstellar bands}

We first discuss the detection and non-detection of DIBs toward five reddened SMC targets, and measure their properties, such as their strength (i.e. equivalent width) and their central or peak absorption velocity/wavelength.

Here we describe in detail the new SMC spectra as well as those reported briefly by Ehrenfreund et al. (2002) and combine our data with the results obtained by Welty et al. (2006) for other sightlines. In the final reduced 3-pixel smoothed data we searched for the following strong and narrow DIBs: $\lambda \lambda 5797$, $5849,6196,6379$ and 6613. In addition we searched for the two strong broad DIB $\lambda \lambda 5780$ and 6284.

In Fig. 3 we show the observed targets for each spectral range covering the seven DIBs at 5780, 5797, 5849, 6196, 6284, 6379 and $6613 \AA$. For comparison we show two Galactic lines-of-sight that are often taken as typical cases for the skin effect. The lineof-sight toward HD 149757 ( $\zeta$ Oph) is characterised as a typical shielded environment, while the line-of-sight toward HD 144217 $\left(\beta^{1} \mathrm{Sco}\right)$ is the prototype of a $\mathrm{UV}$-exposed environment. $\mathrm{AzV} 456$ resembles more a $\zeta$-type Galactic line-of-sight (Sect. 5).

In Table 2 we report the detection of six DIBs in the line of sight toward AzV 456, as well as upper limits for these DIBs toward the other SMC (bar) targets. The equivalent widths $(E W)$ were determined by applying a straight line continuum definition and integration of the DIB profiles. The error in $E W$ is defined as $\sigma(E W)=2 \times F W H M_{\mathrm{DIB}} \times \sigma_{\text {noise }}$, taking $2 \times F W H M_{\mathrm{DIB}}$ as the range of integration and $\sigma_{\text {noise }}$ as a measure of the statistical noise per bin of the adjacent normalised continuum. The upper limit for the $E W$ of non-detected DIBs is $E W_{\text {upper }}=2 \times$ $\sigma_{\text {noise }} \times F W H M_{\mathrm{DIB}}$, where $F W H M_{\mathrm{DIB}}$ is the value measured toward $\mathrm{AzV} 456$ and $\sigma_{\text {noise }}$ is the statistical noise per bin in the corresponding DIB spectral range.

Galactic DIBs correlate well with reddening, i.e. with the dust column density. In Fig. 4 we plot the (upper limit) equivalent widths of the $\lambda \lambda 5797,6196,6379,6613,5780$ and 6284 DIBs versus the SMC reddening (see Sect. 4.2.1). In addition, we include DIB measurements for the sightlines toward HD 62542 (SMC like extinction law; Fig. 2) and the galactic relationships for $\sigma$ and $\zeta$ type sightlines (where available).

Welty et al. (2006) conducted a similar search for DIBs in (different) SMC bar sightlines, and report $5780 \AA$ DIB equivalent widths of about $20 \mathrm{~m} \AA$ toward 5 targets, and $5797 \AA$ DIB equivalent widths of less than $10 \mathrm{~m} \AA$ toward 2 targets. Only for $\mathrm{AzV}$ 476, located well outside the SMC bar, near AzV 488, stronger bands are observed. Equivalent widths are $50 \pm 11 \mathrm{~m} \AA$, $21 \pm 5 \mathrm{~m} \AA$ and $95 \pm 25 \mathrm{~m} \AA$ for the 5780,5797 and $6284 \AA$ DIBs, respectively (see Fig. 4). Per unit reddening, these DIBs are similar in strength to those toward AzV 456. The $6284 \AA$ 

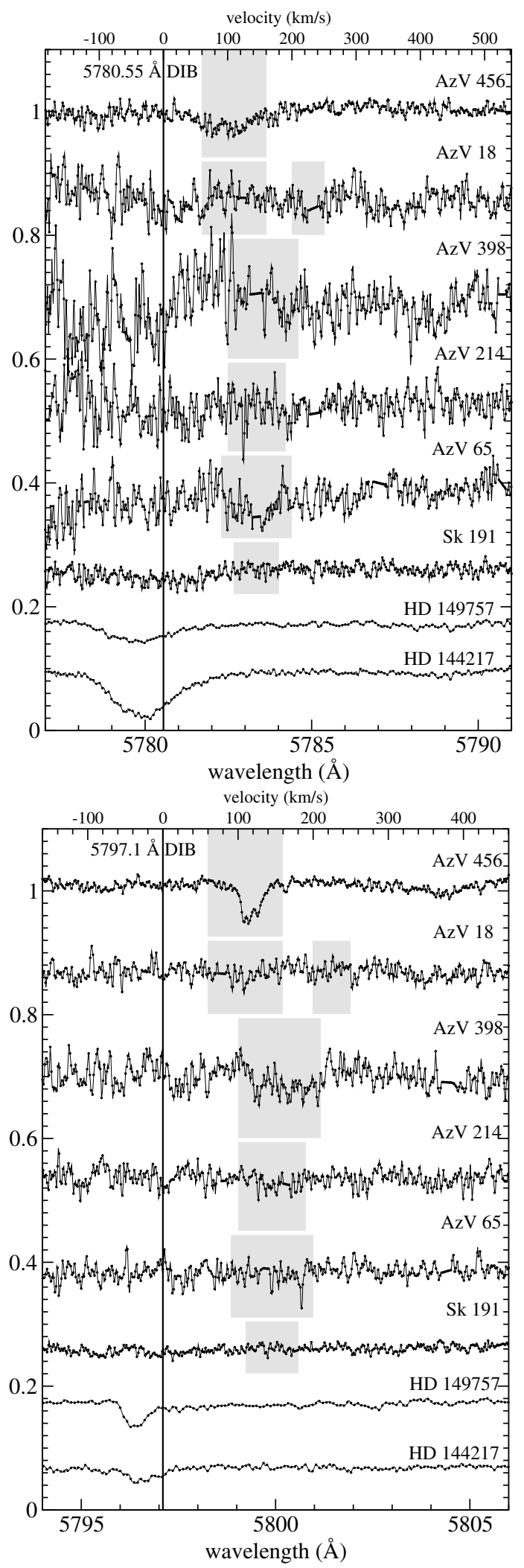

Fig. 3. Normalised spectra of the $5780 \AA$ (top) and $5797 \AA$ (bottom) DIBs observed in the lines-of-sight toward AzV 456, AzV 18, AzV 398, $\mathrm{AzV} 214, \mathrm{AzV} 65$ and Sk 191 (from top to bottom). For comparison the corresponding observed DIB spectra of HD 144217 ( $\sigma$-type) and HD 149757 ( $\zeta$-type) are shown. The shift in wavelength is due to the receding velocity of the SMC and the vertical solid line corresponds to a heliocentric velocity of $0 \mathrm{~km} \mathrm{~s}^{-1}$. The spectra are rebinned to $0.051 \AA$ bin size. The gray boxes indicate the velocity range over which interstellar ionised atomic gas is detected toward each SMC target (from Figs. 5 and 6).
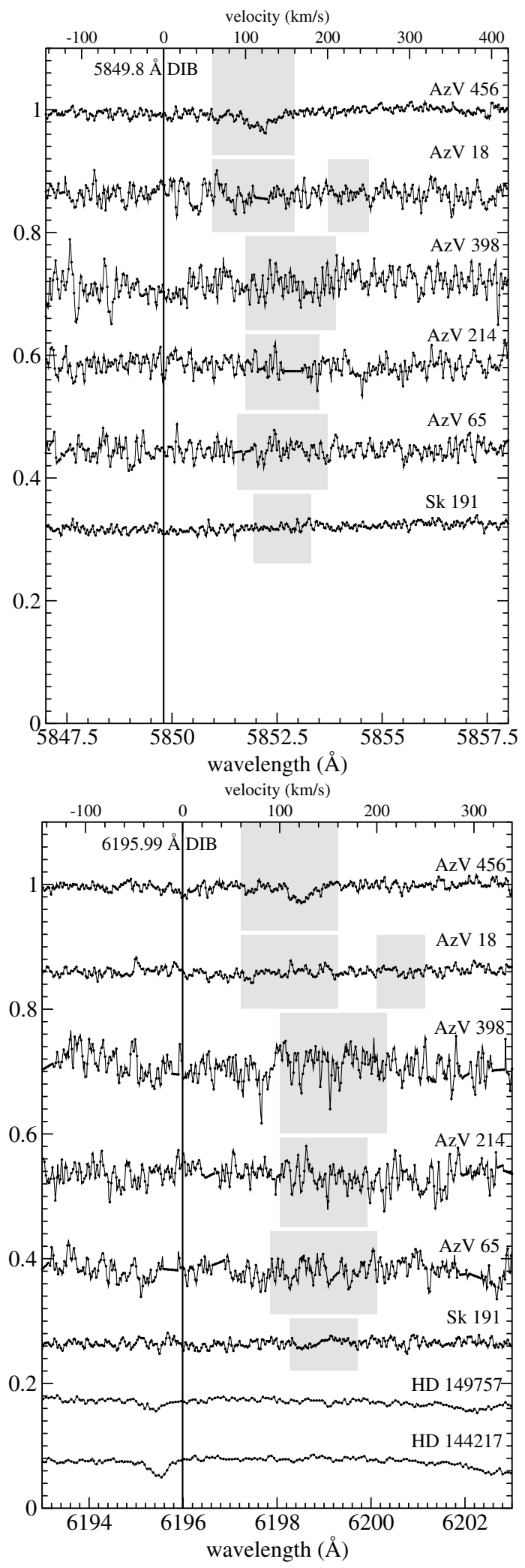

Fig. 3. continued. Normalised spectra of $5849 \AA$ (top) and $6196 \AA$ (bottom) DIBs. 

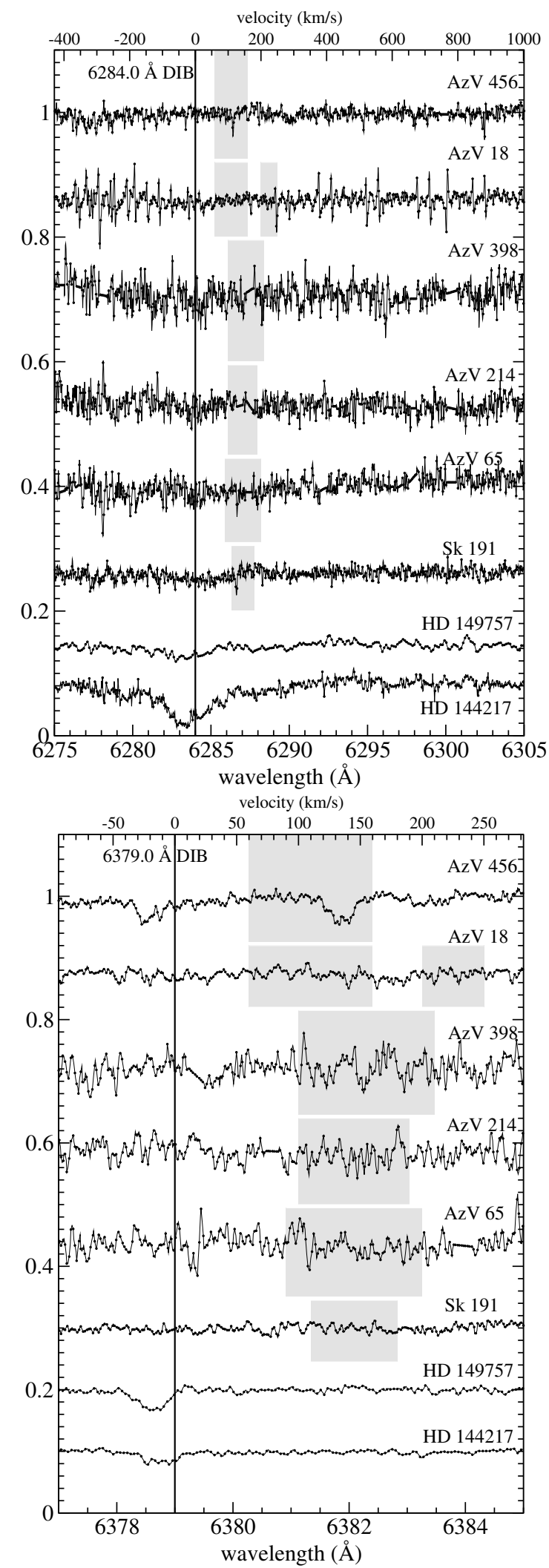

Fig. 3. continued. Normalised spectra of $6284 \AA$ (top) and $6379 \AA$ (bottom) DIBs.

DIB is detected toward AzV 78, situated in the bar, near AzV 18. Measurements of DIBs toward AzV 456 are consistent with our previous results (Ehrenfreund et al. 2002) and those in this work.

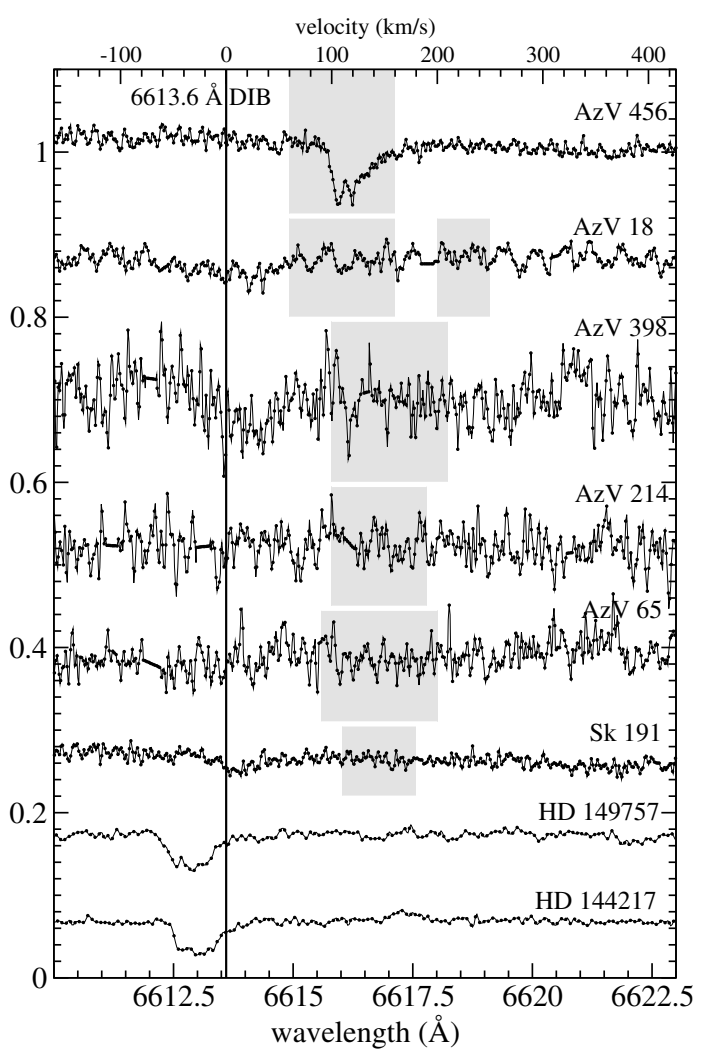

Fig. 3. continued. Normalised spectra of $6613 \AA ̊ ̊ n$ DIBs.

\subsection{Dust}

\subsubsection{Reddening}

The number and size distribution of interstellar dust particles in a line of sight is directly related to the amount of reddening toward the target star. The reddening $E_{B-V}$ of a line-of-sight is defined as the observed colour $(B-V)$ minus the intrinsic colour $(B-V)_{0}$ of the observed star. This requires an accurate determination of the spectral type of the observed star. Recently, the literature has converged to yield a consistent spectral classification of many SMC targets (e.g. the large $2 \mathrm{dF}$ survey of the SMC by Evans et al. 2004b). Since the spectral type is relevant for the determination of the reddening we studied the photospheric spectra. The spectral range between 4000 and $4500 \AA$ was compared with previously published spectra of the same target (e.g. Nandy et al. 1990; Lennon 1997), or if not available, to stellar spectral catalogues (e.g. Evans et al. 2004c). For all spectra we found excellent agreement, and therefore adopted the published spectral types. The often used "stellar" classification spectral range from 4005 to $4500 \AA$ (e.g. Walborn 1977) is shown in Fig. 8 (Online only) for the stars observed in Period 71. In addition, before a comparison between DIB results and SMC reddening can be made, an estimate of the contribution to the total observed $E_{B-V}$ by the foreground Milky Way material is required. The Galactic foreground reddening toward the SMC is estimated to be about $0.05 \pm 0.01$ mag by Bessell (1991) who (re)evaluated previous studies by Azzopardi \& Vigneau (1977), Schwering \& Israel (1989), Walraven \& Walraven (1977), McNamara \& Feltz (1980) and Feast et al. (1960). These values are consistent with the Galactic extinction as derived by Drimmel et al. (2003) in the direction of the SMC, i.e. $E_{B-V}=0.04-0.06 \mathrm{mag}$, as well as with the H I maps by Stanimirović et al. (1999, 2000, 2004). The foreground 


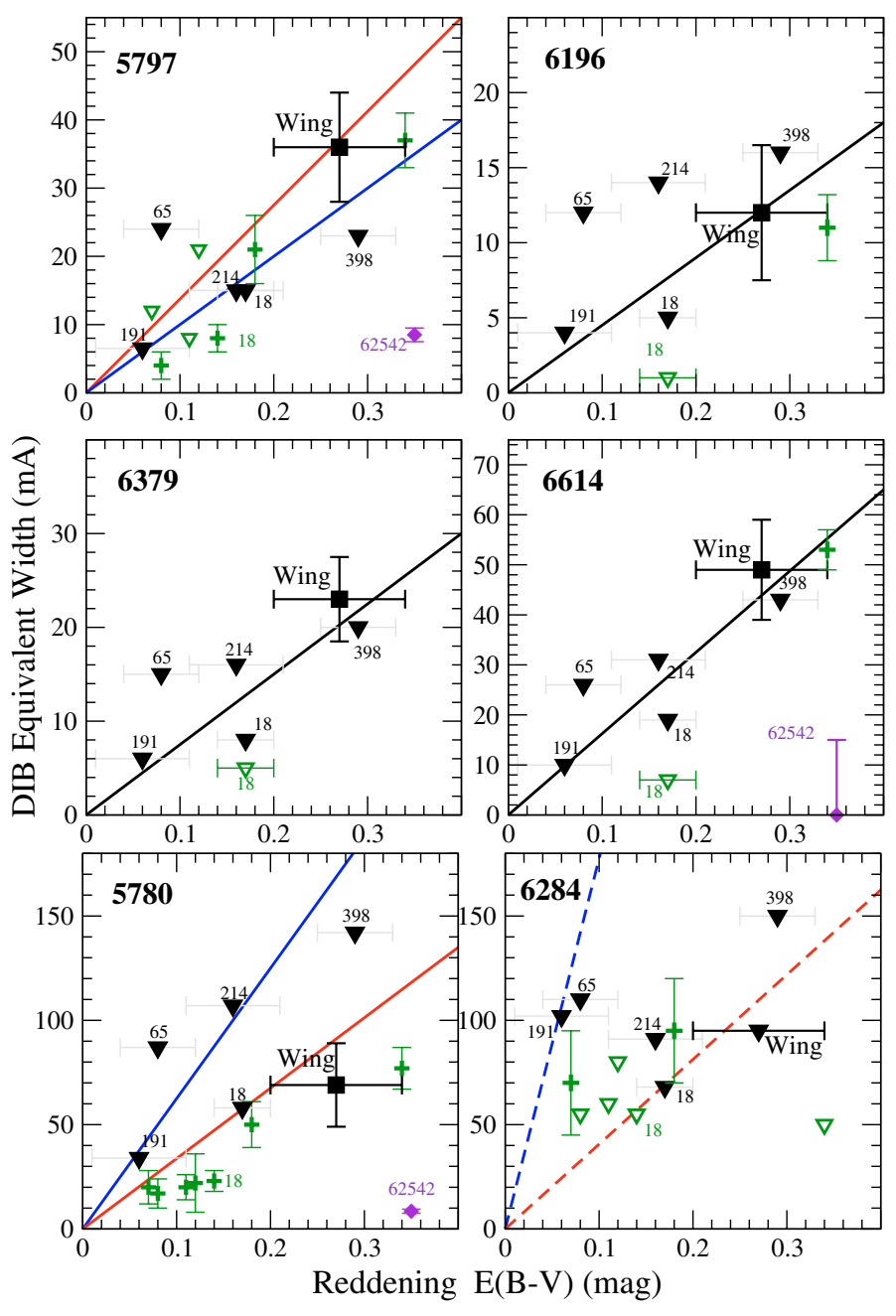

Fig. 4. $\lambda \lambda$ 5797, 6196, 6379, 6614, 5780 and 6284 DIB strengths with error bars for $\mathrm{AzV} 456$ ( $\mathbf{\square}$; this work) and upper limits for DIBs toward the other SMC targets ( $\mathbf{v}$; this work), versus the intrinsic SMC reddening of the corresponding line-of-sight. Upper limits and DIB strengths for lines-of-sight (toward Sk 18, AzV 18, Sk 40, AzV 80, Sk 155 and $\mathrm{AzV}$ 476) obtained by Welty et al. (2006) are indicated with an open downwards triangle (green $\nabla$ ) and a green + , respectively. The solid blue and red lines represent the Galactic relationship between DIB strength and reddening for $\sigma$ and $\zeta$ type lines-of-sight, respectively. The solid black line represents the Galactic relationship between DIB strength and reddening for DIBs that do not show $\sigma / \zeta$ type dependencies. For the $6284 \AA$ DIB dashed blue $(\sigma)$ and red $(\zeta)$ line are based on the DIB strength measured toward HD 144217 and HD 149757, respectively (Table 2). The diamond $(\diamond)$ shows the DIB strengths observed toward the depleted translucent cloud HD $62542\left(E_{B-V}=0.35\right.$ and SMC-like extinction curve). The $\sigma$ and $\zeta$ DIB strength relations reflect the skin-effect (e.g. the destruction of the $\lambda 5797$ DIB and the excitation of the $\lambda 5780$ and 6284 DIB carriers due to a strong effective UV field).

reddening contribution (adopted value $E_{B-V}=0.04 \mathrm{mag}$ ) is then subtracted from the observed reddening for the line-ofsight toward the SMC target. The reddening can also be inferred from the observed atomic sodium column density using $N(\mathrm{Na} \mathrm{I})=1.7 \times E_{B-V}{ }^{1.8}$ (Hobbs 1974a). The resulting values for $E_{B-V}$ are significantly higher than those assumed for the average SMC foreground extinction. The different foreground and SMC reddening estimates are given in Table 1 , with the final adopted $E_{B-V}$ due to the SMC dust given in Table 6 .

\subsubsection{Total-to-selective visual extinction}

For Galactic sightlines the ratio of total-to-selective visual extinction $R_{\mathrm{V}}$ provides a parameter to determine the shape of the extinction curve from the IR to the far-UV. $R_{\mathrm{V}}$ is thus indicative of the size distribution and composition of the interstellar grains in the line-of-sight. $R_{\mathrm{V}}$ can be derived from the infrared colour excess, which is inferred from the observed colours and the intrinsic colours (e.g. Fitzpatrick 1999; Massa \& Fitzpatrick 2003). For four targets $R_{\mathrm{V}}$ values have previously been derived from infrared $J H K$ photometry (Gordon et al. 2003 or Cartledge et al. 2005; Table 6). For the two reddened targets ( $\mathrm{Sk} 191$ and $\mathrm{AzV}$ 65) not included in previous studies the $R_{\mathrm{V}}$ values were computed with $J H K$ photometry from the 2MASS catalogue (Cutri et al. 2003), and subsequent colour excesses (adopting different reference "intrinsic" photometry for corresponding spectral types), applying the relationships derived by Fitzpatrick (1999). Table 7 (online only) gives $R_{\mathrm{V}}$ for different intrinsic colours and spectral types adopted. The final (averaged) $R_{\mathrm{V}}$ for Sk 191 and AzV 65 are given in Table 6. Note that for AzV 65 the scatter is large due to uncertain spectral classification, and skewed toward higher $R_{\mathrm{V}}$. For the remainder of this paper it is assumed that the real extinction curves for these two sightlines are similar to those observed for the other SMC bar lines-of-sight. It is known that the extinction curve derived from IR photometry alone underestimates the real far-UV extinction observed in LMC and SMC lines of sight (Misselt et al. 1999; Gordon et al. 2003). Gordon et al. (2003) argue for a new parameterisation of the ISM characteristics in external galaxies. Therefore, the meaning of $R_{\mathrm{V}}$ (i.e. as a grain size indicator) in the SMC may be different from that in the Milky Way. $R_{\mathrm{V}}$ is used to derive $A_{\mathrm{V}}$ from $E_{B-V}$ (Table 6).

\subsection{Interstellar gas: atomic and diatomic species}

\subsubsection{Column density profiles}

To gain insight into the structure and conditions of the SMC interstellar medium we examined the interstellar line profiles produced by atomic transitions of the gas phase species Ti II, Ca II, Na I, K I and HI. For the respective atoms we constructed apparent column density velocity profiles (Sembach et al. 1993; Savage \& Sembach 1991; Joseph \& Jenkins 1991; Jenkins 1996); we followed an approach similar to that for sightlines observed toward the LMC (Cox et al. 2006). The sodium and calcium column densities were corrected for saturation (Table 3; Cols. 4 and 7). The profiles of the interstellar atomic lines of Ti II, Ca II (H \& K), Na I (D1 \& D2) and K I in the sightline toward AzV 456 are displayed in Fig. 5. The apparent column density profiles for the other sightlines are shown in Fig. 6. Column densities for the total profile can be obtained by simple integration, across the appropriate velocity interval, of the total profile (Tables 3 and 8; Online). A difference between the total column density for the two doublet transitions, if exceeding the statistical error of about 0.02 dex, indicates the presence of saturated components. The column density profiles show that, as expected, the neutral species reside in the narrowest components, while the ionised species are more broadly distributed (in velocity space). The weakly reddened sightlines toward the reference stars AzV 242 and Sk 191 display, as expected, little atomic gas at $\mathrm{SMC}$ velocities.

Figures 5 and 6 show the presence of Galactic foreground material $\left(v \approx 0-50 \mathrm{~km} \mathrm{~s}^{-1}\right)$. Values for Galactic column densities are given in Table 8 (Online only). For some sightlines 
Table 2. Diffuse interstellar bands investigated in this study. The $E W$ and associated statistical error $\sigma_{E W}$ (derived from the adjacent continuum) is quoted in $\mathrm{m} \AA$ (first part, Col. 5). Observed central wavelengths, heliocentric velocities and $F W H M$ are given in Cols. 2-4, respectively. For non-detected DIBs upper limits are calculated adopting $E W_{\text {upper limit }}=2 \sigma_{\text {noise }} F W H M_{\text {DIB }}$, with $\sigma_{\text {noise }}$ the noise in the spectral range of the expected DIB, and $F W H M_{\text {DIB }}$ the full width at half maximum of the corresponding DIB observed toward AzV 456 . For AzV 18 the first two values are derived from spectra obtained in 2001 and 2003, respectively. The third value is obtained from Welty et al. (2006). Our DIB equivalent widths for $\mathrm{AzV} 456$ are consistent with those derived by Welty et al. (2006). The reported high upper limits for the 5780 $\AA$ are due to the lower signal-tonoise in the spectral range and its larger $F W H M$. The DIB spectral ranges are plotted in Fig. 3 . The $E_{B-V}$ values were derived by subtracting the Milky Way foreground contamination from the line-of-sight reddening (see Sect. 4.2.1 and Table 1).

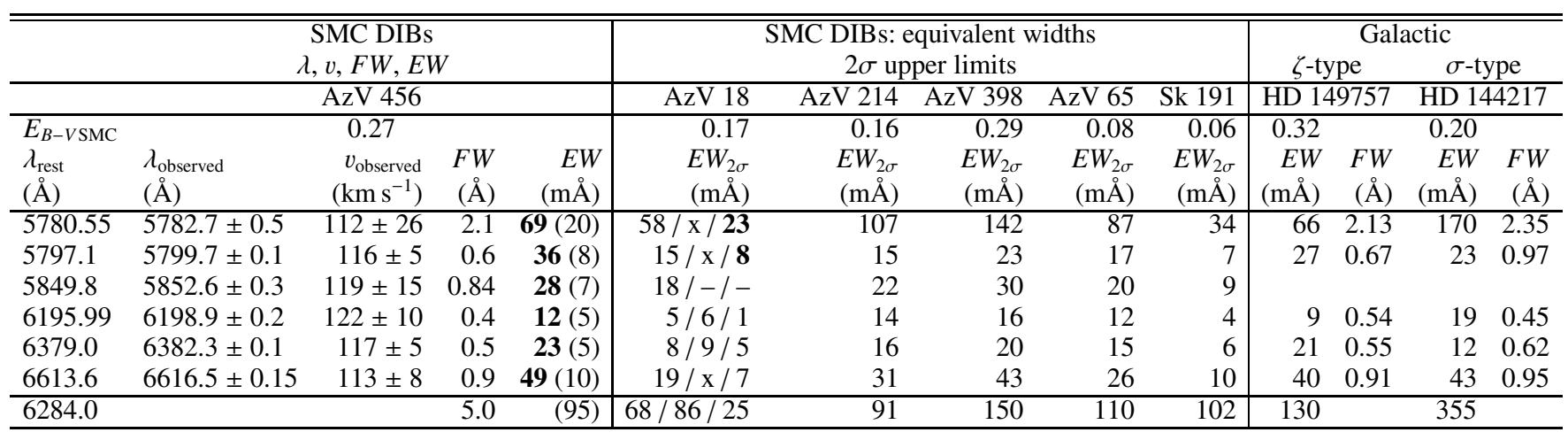

this could cause significant foreground reddening as discussed in Sect. 4.2.1.

Neutral hydrogen $21 \mathrm{~cm}$ profiles are shown in the bottom panels of Figs. 5 and 6 . H I column density profiles were retrieved from the SMC ATCA+Parkes Magellanic Cloud survey (Stanimirović et al. 1999; Kim et al. 2003; Staveley-Smith et al. 1997, 2003). These profiles nicely show the "2-component" SMC structure as visualised by Stanimirović et al. (2004). When comparing the $21 \mathrm{~cm}$ neutral hydrogen velocity profile with that of the other atomic species it is important to note that it is not possible to discern between material (with a same radial velocity) situated in front or behind the observed star. Therefore, the neutral hydrogen in the line-of-sight corresponding to the atomic species is, in principle, more accurately measured in the Ly $\alpha$ line, than through the $21 \mathrm{~cm}$ radio velocity profile. Thus for calculation of the gas-to-dust ratios we use $N(\mathrm{HI})$ and $A_{\mathrm{V}}$ derived by Gordon et al. (2003) and Cartledge et al. (2005) (Table 4). For the two targets with no available Ly $\alpha$ profiles we adopt the $21 \mathrm{~cm} \mathrm{H}$ I column density (intergrated over the SMC ISM velocity range), corrected for beam filling by a factor of $0.75 \pm 0.25$ (Table 4; see Danforth et al. 2002). Table 4 also lists for comparison other literature values for $N(\mathrm{HI})$ (Bouchet et al. 1985; Fitzpatrick 1985; and Evans et al. 2004a) and for $N\left(H_{2}\right)$ (Cartledge et al. 2005).

\subsubsection{Weak (di)atomic transitions toward AzV 456}

In addition to the diffuse interstellar bands the line-of-sight toward $\mathrm{AzV} 456$ also reveals the $\mathrm{CH}$ molecule transition at $4300.3 \AA$, and the $\mathrm{Ca}$ I line at $4226.7 \AA$, for which we derive column densities $\log N=13.40$ and $\lesssim 9.85 \mathrm{~cm}^{-2}$, respectively. The $\mathrm{CH}^{+}$(4232.548 $\AA$ ) line is not detected, yielding an upper limit of $\log N \lesssim 12.05 \mathrm{~cm}^{-2}$ (Table 5; Fig. 7). In addition, we show the transitions of the K I UV doublet (4044.136, 4047.206 $\AA$ ) and the Na I UV doublet $(3302.37,3302.98 \AA)$. From the K I UV line equivalent width we derive $\log N(\mathrm{KI})=12.7$ and $12.6 \mathrm{~cm}^{-2}$, respectively (Table 5). The Na I UV lines yield $\log N(\mathrm{NaI})=$ 13.4 and $13.6 \mathrm{~cm}^{-2}$, respectively (Table 5).
Table 3. Small Magellanic Cloud atomic column densities for Na I, Ca II and Ti II derived via the construction of apparent column density velocity profiles and subsequent line profile integration $\left(v \approx 80-200 \mathrm{~km} \mathrm{~s}^{-1}\right)$. The error in $\log N$ is 0.02 dex for Ti II and Ca II K, and $\approx 0.03-0.04$ dex for the more saturation sensitive Na I D line. Line transition wavelengths and oscillator strengths are adopted from Morton (2003). For both Na I and $\mathrm{Ca}$ II the individual doublet line column densities are given, as well as the "optical depth - velocity" corrected column densities (see text for details).

\begin{tabular}{|c|c|c|c|c|c|c|c|}
\hline & \multicolumn{7}{|c|}{ Column density $\log N\left(\mathrm{~cm}^{-2}\right)$} \\
\hline & \multicolumn{3}{|c|}{$\mathrm{NaI}$} & \multicolumn{3}{|c|}{ CaII } & \multirow[t]{2}{*}{ Ti II } \\
\hline & D1 & $\mathrm{D} 2$ & corr & $\bar{K}$ & $H$ & corr & \\
\hline $\mathrm{AzV} 456$ & 12.41 & 12.17 & 12.98 & 12.34 & 12.51 & 12.73 & 12.39 \\
\hline AzV 18 & 12.63 & 12.48 & 12.72 & 12.94 & 12.92 & 12.94 & 12.95 \\
\hline AzV 214 & 12.73 & 12.55 & 12.95 & 12.81 & 12.90 & 12.97 & 12.86 \\
\hline AzV 398 & 12.88 & 12.69 & 13.17 & 13.17 & 13.14 & 13.17 & 13.16 \\
\hline AzV 65 & 12.37 & 12.27 & 12.48 & 13.06 & 13.16 & 13.25 & 13.24 \\
\hline Sk 191 & 12.28 & 11.98 & $\gg 12.3$ & 12.19 & 12.23 & 12.24 & 12.17 \\
\hline AzV 242 & 12.09 & 12.01 & 12.17 & 12.72 & 12.74 & 12.74 & 12.64 \\
\hline
\end{tabular}

These values clearly indicate the strong saturation of the $\mathrm{Na}$ I D doublet lines. We searched the red spectra of $\mathrm{AzV} 18$ for $C_{2}$ Philips (2-0) bands in the spectral range 8750-8780 , but these lines are too weak to detect if present. The presence of diatomic molecules is indicative of a complex chemistry in the diffuse medium toward AzV 456.

\subsubsection{Atomic line ratios}

The Routly-Spitzer effect (Routly \& Spitzer 1952; Siluk \& Silk 1974) predicts that the relative amount of $\mathrm{Na}$ I with respect to the amount of $\mathrm{Ca}$ II, in the gas phase, provides information on the amount of $\mathrm{Ca}$ II liberated from dust grains into the gas phase. In this scheme a lower ratio arises in a more disruptive/turbulent environment with increased grain destruction. On the other hand, a higher ratio is evidence for a more quiescent environment. From the $N(\mathrm{Na} \mathrm{I}) / N(\mathrm{Ca}$ II) ratios observed in this work (Table 6) we conclude that, given the above presumption, the cloud in the line-of-sight toward $\mathrm{AzV} 456$ is (relatively) quiescent, and the 


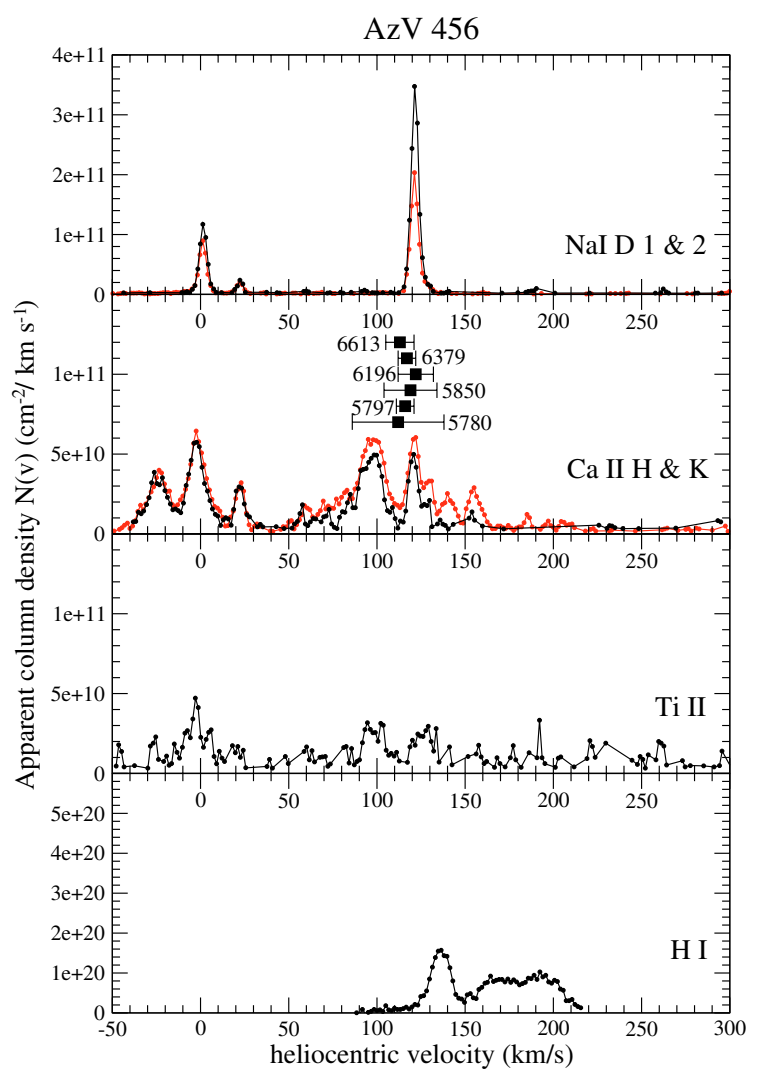

Fig. 5. Line profiles (in heliocentric velocity space) for the transitions of Ca II H\&K, Ti II, Na I D and H I for the line-of-sight toward AzV 456. For AzV $456 \mathrm{McGee} \&$ Newton (1986) identified 3 components at 134, 173 and $190 \mathrm{~km} \mathrm{~s}^{-1}$, confirmed by these profiles. Central peak velocities for DIBs observed in AzV 456 are shown at the top of the second panel. DIB carrier velocities are only marginally lower than those of $\mathrm{CH}$ and the neutral species (Na I and K I) at $\sim 123 \mathrm{~km} \mathrm{~s}^{-1}$ (see Table 5 and Fig. 7). Note the very weak Ti II and weak Ca II profile.

Table 4. Small Magellanic Cloud atomic hydrogen and molecular hydrogen column densities from different sources.

\begin{tabular}{lcccccc}
\hline \hline \multicolumn{5}{c}{ H I column density $\log N(\mathrm{HI}) \mathrm{cm}^{-2}$} & \multicolumn{1}{l}{$\log N\left(\mathrm{H}_{2}\right) \mathrm{cm}^{-2}$} \\
& {$[1]$} & {$[2]$} & {$[3]$} & {$[4]$} & {$[5]$} & {$[6]$} \\
\hline AzV 456 & $21.60 \pm 0.05$ & 21.63 & $21.4 \pm 0.1$ & 21.18 & $20.93(0.09)$ \\
AzV 18 & $21.93 \pm 0.02$ & 22.10 & $22.0 \pm 0.1$ & 21.95 & 21.80 & $20.36(0.07)$ \\
AzV 214 & $21.40 \pm 0.08$ & 21.81 & $21.9 \pm 0.1$ & & & \\
AzV 398 & $21.90 \pm 0.10$ & 21.85 & $22.2 \pm 0.1$ & \multicolumn{2}{c}{} \\
AzV 65 & & 22.01 & & \multicolumn{2}{|c}{21.40} \\
Sk 191 & - & $21.6 \pm 0.1$ & \multicolumn{2}{c}{$17.21_{-1.25}^{+1.00}$} \\
AzV 242 & & 21.59 & $21.5 \pm 0.1$ & & \\
\hline
\end{tabular}

[1] Gordon et al. (2003) (via Ly $\alpha$ ); [2] H I ATCA+PARKES survey (Stanimirović et al. 2000, 2004; Staveley-Smith et al. 1997), see Sect. 4.3 and Fig. 5 (HI $21 \mathrm{~cm}$ emission); [3] Bouchet et al. (1985); [4] Fitzpatrick (1985) (via Ly $\alpha$ ); [6] Cartledge et al. (2005).

clouds toward $\mathrm{AzV} 18$ and $\mathrm{AzV} 65$ show a higher degree of grain disruption.

The depletion of the refractory titanium ion, $\delta_{\mathrm{Ti}}$ (Table 6), from the gas phase (onto the dust grains) is highest for $\mathrm{AzV} 456$ and Sk 191, in line with the previous statement.

This conclusion is further substantiated by the absence of $\mathrm{CH}^{+}$toward $\mathrm{AzV}$ 456. $\mathrm{CH}^{+}$is very abunddant in the Milky Way due to endoergic reactions driven by shocks and/or turbulent

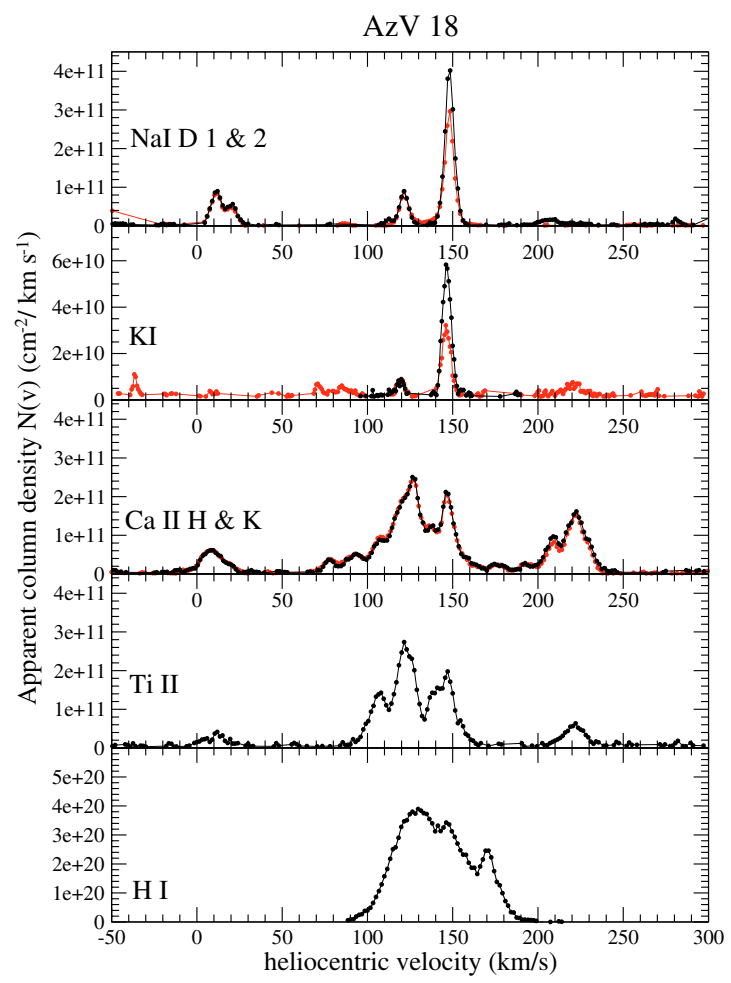

AzV 398

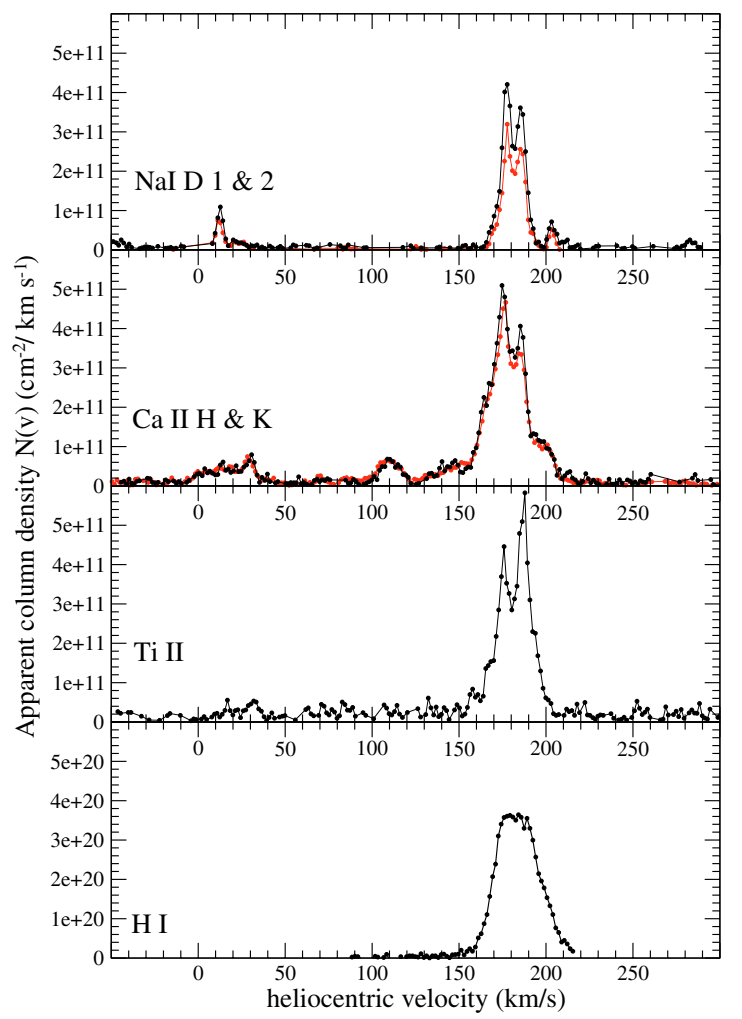

Fig. 6. Line profiles (in heliocentric velocity space) for the transitions of NaID, CaII H\&K, Ti II and H I for the lines-of-sight toward AzV 18 and AzV 398. The KI is shown for AzV 18, whilst not available for AzV 398. The lines-of-sight toward AzV 398 probes mainly the SMC component " 2 ", at the higher velocity, of the SMC diffuse medium. The Ca II and Ti II gas (both with very similar profiles) toward $\mathrm{AzV} 398$ is not as broadly distributed in velocity space as seen toward AzV 456 and AzV 18, consistent with the narrower H I profile. The "high" velocity cloud (200-250 $\left.\mathrm{km} \mathrm{s}^{-1}\right)$ towards AzV 18 is not included in the reported total column densities. Details on the construction of these profiles are in Sect. 4.3. 

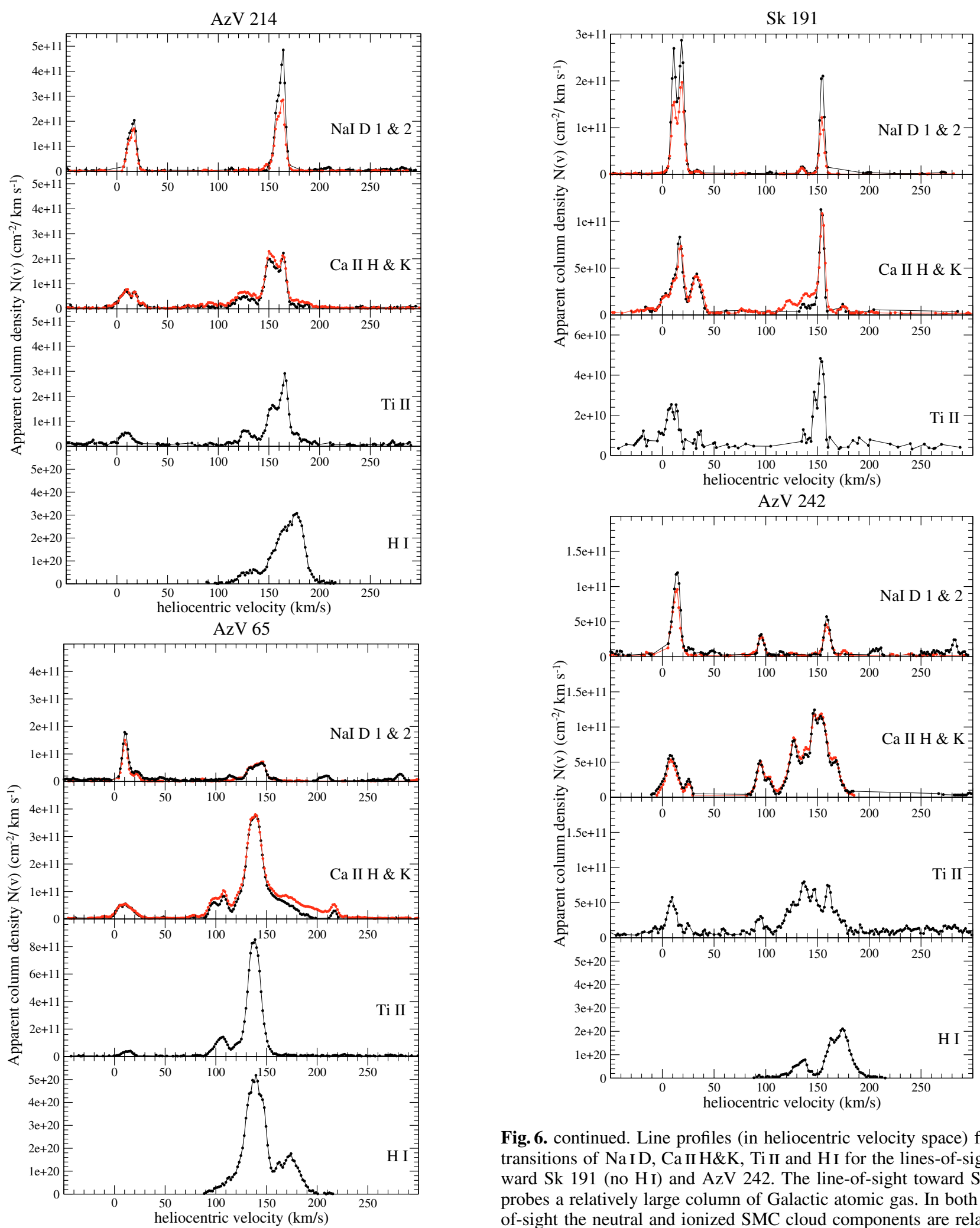

Fig. 6. continued. Line profiles (in heliocentric velocity space) for the transitions of $\mathrm{NaID}$, Ca II H\&K, Ti II and H I for the lines-of-sight toward AzV 214 and AzV 65. The lines-of-sight toward AzV 214 also probes mainly the SMC component " 2 ", at the higher velocity, of the SMC diffuse medium. The Ca II and Ti II gas (both with very similar profiles) is not as broadly distributed in velocity space as seen toward $\mathrm{AzV} 456$ and $\mathrm{AzV}$ 18, consistent with the narrower $\mathrm{H}$ I profile. The lineof-sight toward AzV 65 shows a very weak $\mathrm{Na}$ I line, while the H I, Ca II and Ti II lines are strong (also compared to the other lines-of-sight). Details on the construction of these profiles are in Sect. 4.3. 
Table 5. Weak diatomic and atomic UV lines: $\mathrm{CH}, \mathrm{CH}^{+}, \mathrm{Ca}$ I, $\mathrm{KI}$ and $\mathrm{Na}$ I toward AzV 456. The spectral lines are shown in Fig. 7. Central velocities $v\left(\mathrm{~km} \mathrm{~s}^{-1}\right)$, equivalent widths $E W(\mathrm{~m} \AA)$ and column densities $\log N\left(\mathrm{~cm}^{-2}\right)$ are listed.

\begin{tabular}{llllll}
\hline \hline Species & $\begin{array}{l}\lambda_{\text {rest }} \\
(\AA)\end{array}$ & $f$ & $\begin{array}{l}v \\
\left(\mathrm{~km} \mathrm{~s}^{-1}\right)\end{array}$ & $\begin{array}{l}E W \\
(\mathrm{~m} \AA)\end{array}$ & $\begin{array}{l}\log N^{4} \\
\left(\mathrm{~cm}^{-2}\right)\end{array}$ \\
\hline $\mathrm{CH}$ & 4300.303 & $0.0053^{3}$ & 123 & 22.0 & 13.40 \\
$\mathrm{CH}^{+}$ & 4232.548 & $0.0056^{3}$ & & $\lesssim 1$ & $\lesssim 12.05$ \\
$\mathrm{Ca} \mathrm{I}_{\mathrm{K}}$ & 4226.728 & $1.77^{1}$ & 123 & $\lesssim 2$ & $\lesssim 9.85$ \\
& 4044.142 & $0.00608^{2}$ & 126 & 4.6 & 12.72 \\
$\mathrm{Na}$ & 4047.213 & $0.00305^{2}$ & 121 & 1.7 & 12.58 \\
& 3302.37 & $0.00900^{3}$ & 122 & 23.0 & 13.42 \\
& 3302.98 & $0.00446^{3}$ & 123 & 16.5 & 13.58 \\
\hline
\end{tabular}

${ }^{1}$ G. Risberg (1968); ${ }^{2}$ NIST Atomic spectra database (P. Risberg, 1956); ${ }^{3}$ Morton (2003); ${ }^{4}$ Welty et al. (2006) find $\log N=13.60, \lesssim 12.40$ and 12.47 for $\mathrm{CH}, \mathrm{CH}^{+}$and $\mathrm{CN}$, respectively.

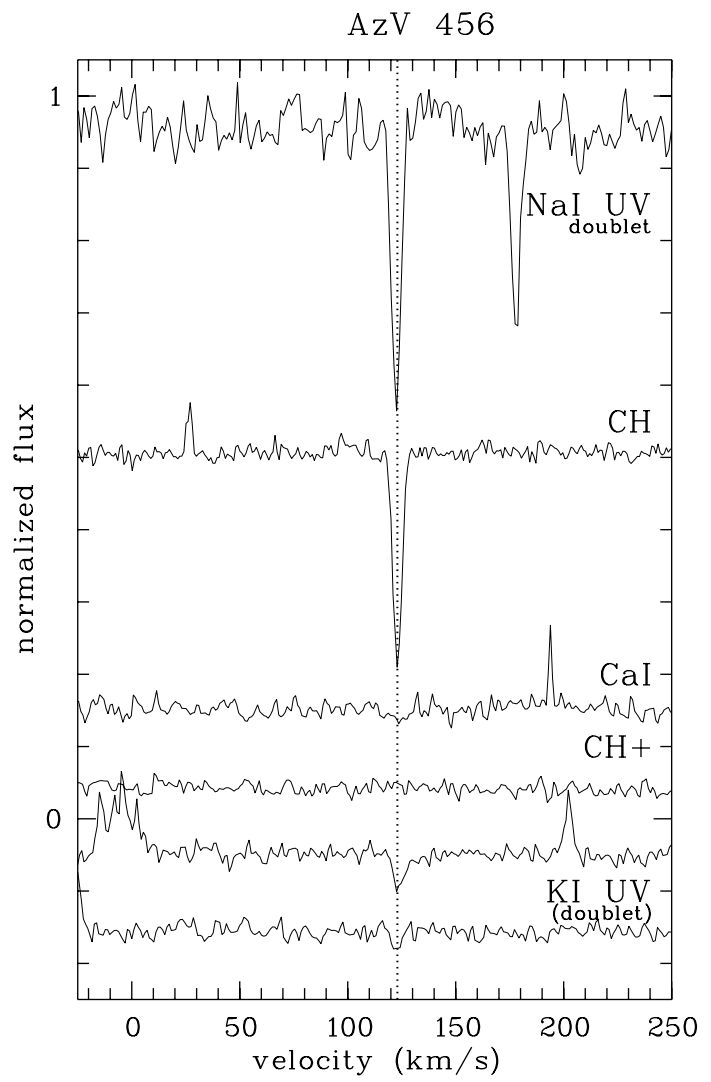

Fig. 7. Absorption lines (in heliocentric velocity space) for the transitions of the diatomic molecules $\mathrm{CH}(4300 \AA)$ and $\mathrm{CH}^{+}(4236 \AA)$ and the weak atomic UV lines of Ca I (4226.7 $⿱$ ), K I (4044.1, 4047.2 $\AA$ ) and $\mathrm{Na}$ I $(3302.37,3302.98 \AA)$ toward AzV 456. Spectra are normalised to unity and, for clarity, shifted on $y$-axis.

dissipation (Falgarone et al. 1995; Spaans 1995) and its absence is only expected for quiescent gas.

Previous studies show that the relative, though not the absolute, depletion levels of heavy elements are constant throughout the SMC (see Sect. 5). Since Ti II is the dominant ion in the diffuse ISM $\left(\mathrm{IP}_{\mathrm{Ti}}=6.828 \mathrm{eV}\right.$ and $\left.\mathrm{IP}_{\mathrm{Ti} \text { II }}=13.576 \mathrm{eV}\right)$ a change in the ratio $N(\mathrm{Ti}$ II $) / N(\mathrm{Ca}$ II $)$ is then caused mainly by a change in the ratio $N(\mathrm{Ca}$ II $) / N(\mathrm{Ca}$ III $)$ (i.e. the ionization level; $\mathrm{IP}_{\mathrm{CaI}}=$ $6.113 \mathrm{eV}$ and $\left.\mathrm{IP}_{\mathrm{CaI}}=11.872 \mathrm{eV}\right)$, the relative total levels of titanium and calcium being equal, which is directly governed by the strength (i.e. total energy flux of UV photons) and/or the hardness (the ratio of high versus low energy photons in the UV field) of the UV radiation field. Thus, a higher ratio of Ti II over Ca II can indicate a stronger UV radiation field. Hunter et al. (2006) found $N(\mathrm{Ti}$ II $) / N(\mathrm{Ca}$ II $) \sim 0.3$ with little variation over a large range of distances and column densities, consistent with the calcium ionisation balance controlled by the ISRF. The SMC ratios we find are substantially higher $(\sim 0.8-1.0$; Table 6$)$, except for $\mathrm{AzV} 456(\sim 0.45)$, thus indicating a shift in the ionisation balance due to the stronger ISRF observed for the SMC. For the observed targets this suggests that the effective UV fields toward AzV 18, AzV 65, Sk 191 and AzV 214 are similar, and somewhat stronger than those toward AzV 398 and AzV 456 (Table 6).

Thus a quiescent, shielded environment exists in the ISM toward AzV 456 and Sk 191, whereas a more turbulent, UV exposed environment is observed for the SMC bar ISM.

\section{Interstellar gas, dust and diffuse bands in the SMC}

The SMC wing has a modest star formation rate (weaker $\mathrm{H} \alpha$ emission), and is more protected from the harsh UV field than the diffuse medium in the SMC bar, an active star formation region. The average interstellar radiation field (ISRF) inferred for the SMC is 4 to 10 times stronger (in units of the standard ISRF) than that of the Solar neighbourhood (Lequeux 1979; Vangioni-Flam et al. 1980; Azzopardi et al. 1988; Bot et al. 2004). The molecular hydrogen fractions and $\mathrm{H}_{2}$ level populations observed for SMC \& LMC diffuse sightlines (by e.g. FUSE) are, on average, in line with an $I S R F \geq 10$ times the standard (Galactic) ISRF (Browning et al. 2003; Lee et al. 2007). Welty et al. (2006) derive, via $\mathrm{HI}$ and $\mathrm{H}_{2}$, an enhancement of the ISRF of $\sim 28-83$ for the lines-of-sight near the star-forming (H II) regions in the southwest end of the SMC bar (AzV 18, Sk 18), but find a normal Galaxy-like value, $\sim 0.6$, for the sightline toward AzV 456.

Another difference between Galactic, SMC wing and SMC bar sightlines are their respective gas-to-dust ratios. The atomic gas-to-dust ratio (approximated by $N(\mathrm{HI}) / A_{\mathrm{V}}$ or $N(\mathrm{HI}) / E_{B-V}$; Table 6$)$ is an indicator of the level of dust shielding. If possible, it is more accurate to include $\mathrm{H}_{2}$ to obtain the total gas-to-dust ratio $N\left(H_{\text {total }}\right) / A_{\mathrm{V}}$, with $N\left(H_{\text {total }}\right)=$ $N(\mathrm{H} \mathrm{I})+2 N\left(\mathrm{H}_{2}\right)$ (Table 9; Online only). On a galaxy-wide scale the gas-to-dust ratio is a good indicator of metallicity (e.g. Issa et al. 1990). The gas-to-dust ratio $\left(N(\mathrm{HI}) / A_{\mathrm{V}}\right)$ is about ten times higher in the SMC (dust-to-gas factor ten times lower), with the exception of $\mathrm{AzV} 456$ for which the gas-to-dust ratio is only two to three times higher. The SMC gas-to-dust ratio inferred from $170 \mu \mathrm{m}$ and $\mathrm{HI}(21 \mathrm{~cm})$ maps is about 30 times that of the Galactic gas-to-dust ratio (Bot et al. 2004). Gordon et al. (2003) found that if $N(\mathrm{HI}) / A_{\mathrm{V}}$ decreases, the bump-strength increases and the UV rise decreases. The variation in the gas-to-dust ratio may thus reflect a change in balance of dust destruction and formation processes (accretion on dust grains) between the diffuse medium and denser/molecular clouds. This may also have affected the DIB carriers. The integrated gas-to-dust ratio depends on the relative fraction of matter in diffuse/dense components and on the supernova rate, and thereby on the star formation history (e.g. Hirashita 1999).

The depletion level of heavy elements in SMC dust is lower than expected from the overall metallicity (Dufour 1984; 
Table 6. Reddening, total-to-selective extinction, visual extinction, atomic column density ratios, and gas-to-dust ratios for sightlines in the SMC. A higher Na I/Ca II ratio implies less Ca II liberated from dust grains by destructive processes and thus a more quiescent environment. A lower ratio thus indicates a more turbulent medium where dust grains are destroyed and $\mathrm{Ca}$ II is released into the gas phase. The Ti depletion level $\delta_{\mathrm{Ti}}$ gives a measure of the lock up of the refractory ion in grains. A higher Ti II/Ca II ratio indicates a stronger/harder UV field. $E_{B-V}$ is corrected for foreground reddening. $R_{\mathrm{V}}$ for $\mathrm{AzV} 65$ and Sk 191 from Table 7, other values for $R_{\mathrm{V}}$ (and $A_{\mathrm{V}}$ ) from Gordon et al. (2003).

\begin{tabular}{lllllll}
\hline \hline ID & AzV 456 & AzV 18 & AzV 214 & AzV 398 & AzV 65 & Sk 191 \\
\hline$E_{B-V}$ (mag) [1] & $0.30 \pm 0.03$ & $0.17 \pm 0.01$ & $0.16 \pm 0.01$ & $0.23 \pm 0.02$ & & \\
$E_{B-V}$ (mag) [Sect.4.2] & $0.27 \pm 0.07$ & $0.17 \pm 0.03$ & $0.16 \pm 0.05$ & $0.29 \pm 0.04$ & $0.08 \pm 0.04$ & $0.06 \pm 0.05$ \\
$R_{\mathrm{V}}[1]$ & $2.05 \pm 0.17$ & $3.30 \pm 0.38$ & $2.40 \pm 0.30$ & $3.14 \pm 0.34$ & & \\
$R_{\mathrm{V}}[2]$ & $2.19 \pm 0.23$ & $2.90 \pm 0.42$ & & & $\sim 2.8$ & $\sim 2.7$ \\
$A_{\mathrm{V}}[1]$ & $0.54 \pm 0.06$ & $0.55 \pm 0.08$ & $0.35 \pm 0.05$ & $0.68 \pm 0.11$ & $\sim 0.22$ & $\sim 0.16$ \\
$A_{\mathrm{V}}[2]$ & $0.57 \pm 0.08$ & $0.49 \pm 0.11$ & & & & \\
\hline & & & & & & - \\
$N(\mathrm{H} \mathrm{I}) / A_{\mathrm{V}}\left(\times 10^{21}\right)[1]$ & $7.40 \pm 1.20$ & $15.45 \pm 2.33$ & $7.08 \pm 1.77$ & $11.69 \pm 3.43$ & - & 15.7 \\
$N(\mathrm{H} \mathrm{I}) / A_{\mathrm{V}}\left(\times 10^{21}\right)[3]$ & 7.94 & 22.73 & 18.60 & 10.53 & 46 & $>1.15$ \\
$\mathrm{Na} / \mathrm{Ca}$ II & $1.8 / 7.1(\mathrm{uv})$ & 0.60 & 0.95 & 1.0 & 0.17 & 0.85 \\
$\mathrm{Ti} \mathrm{II} / \mathrm{Ca}$ II & 0.45 & 1.02 & 0.98 & 0.78 & 0.98 & -9.4 \\
$\log N(\mathrm{Ti}) / N(\mathrm{H})$ & -9.4 & -9.0 & -8.5 & -8.7 & -8.8 & -1.9 \\
$\log \delta_{\mathrm{Ti}}[4]$ & -2.5 & -2.1 & -1.6 & -1.8 & -1.9 & -2.5 \\
\hline
\end{tabular}

[1] Gordon et al. (2003); [2] Cartledge et al. (2005); [3] H I ATCA+PARKES survey (Stanimirović et al. 2004, 2000, 1999; Staveley-Smith et al. 1997, see Sect. 4.3; [4] $\log (\mathrm{Ti} / \mathrm{H})_{\odot}=-6.9$ (Asplund et al. 2005). $\log \delta_{\mathrm{Ti}}=\log [N(\mathrm{Ti}) / N(\mathrm{H})]-\log (\mathrm{Ti} / \mathrm{H})_{\odot}$.

Sauvage \& Vigroux 1991). Welty et al. (2001) suggest that Si and $\mathrm{Mg}$ are undepleted in the SMC. Conversely, Sofia et al. (2006) find that these elements are depleted from the SMC ISM by similar levels in both the wing and bar regions, but the levels of depletion were not well determined. The depletion patterns appear similar to those observed in the Galaxy (e.g. Vladilo 2002). Unfortunately, depletion levels for interstellar carbon are currently not available. However, Prevot et al. (1984) find that in low metallicity environments, like the SMC, the number density of carbon stars is high. Therefore, the abundance of carbon rich stardust could be higher than suggested from other metallicity indicators, such as for example, H II regions. On the other hand, the high SMC gas-to-dust ratio (10-30 times Galactic; see above) points toward a much more severe deficiency of PAHs than expected solely from the lower heavy element abundance. Except for those regions, like the SMC wing, with a lower gas-to-dust ratio and therefore, through its inverse proportionality, higher metallicity $\left(Z_{\text {wing }} \approx 0.25 Z_{\odot}\right)$. Of course, the fraction of carbon locked-up in PAHs can be different for the SMC and Galaxy.

A lower dust and carbon abundance, and hence lower $\mathrm{H}_{2}$ formation rate together with a stronger UV field may cause $\mathrm{H}$ I to dominate over $\mathrm{H}_{2}$ even in regions with higher density (compared to the MW). High $\mathrm{H}_{2}$ density SMC sightlines likely exhibit $\mathrm{H}_{2}$ formation rates closer to the Galactic value (Lee et al. 2007), while low density SMC sightlines are expected to have formation rate reduced by factor ten. This suggests that in the Magellanic Clouds H I absorption traces not only diffuse, but also denser clouds, which remain atomic. Indeed, from the FUSE survey of the SMC a total diffuse $\mathrm{H}_{2}$ mass of $M\left(\mathrm{H}_{2}\right)=$ $2 \times 10^{6} M_{\odot}$ is found, which is less than $2 \%$ of the H I mass in the clouds (Thorburn et al. 2003). This implies a low overall molecular content, high star formation efficiency and/or substantial molecular mass in cold, dense clouds that are not probed by the FUSE survey. In addition, the low $\mathrm{H}_{2}$ fraction (and thus $\mathrm{H}_{2}$ formation rate) in the SMC bar might also be directly connected to the lack of diatomic molecules and $2175 \AA$ A bump and DIB carriers, as opposed to the SMC wing, which has a high $\mathrm{H}_{2}$ fraction, where diatomic molecules, $2175 \AA$ bump and DIB carriers are clearly present.

DIBs and diatomics are also present in the line of sight toward AzV 476 (Welty et al. 2006), which is also located outside the bar and shows a high $\mathrm{H}_{2}$ column density and less strong, 2.1 times average Galactic, ISRF. However, no extinction curve/2175 A bump information is available for this line of sight.

From Fig. 4 and Table 9 we see that the DIB strengths measured toward $\mathrm{AzV} 456$ are similar, with respect to reddening, to those of a Galactic $\zeta$ type environment. AzV 456 is also the only SMC line-of-sight that shows Galactic-like dust extinction (both a bump and a weak far-UV extinction). For the SMC bar line-ofsight toward AzV 18, weak 5797 and 5780 Å DIBs are detected and upper limits derived for the DIBs at 6196, 6284, 6379 and $6614 \AA$. These values show that the DIBs are weak per unit reddening compared to the Galactic relation. Sightlines with very weak DIBs show a similar extinction curve with no bump and a strong far-UV rise (Cardelli \& Savage 1988; Fig. 2). For example, the Galactic target HD 62542 (Sect. 3) shows no bump and weak DIBs but it does have a high $\mathrm{H}_{2}$ fraction, just as observed for $\mathrm{AzV}$ 456. The lack of DIBs in HD 62542 shows that their carrier chemistry is not directly related to "standard" diffuse ISM chemical pathways related to $\mathrm{H}_{2}$. The inferred underabundance of the $6284 \AA$ DIB carrier toward AzV 456 is also seen toward the LMC 30 Doradus target Sk-68 135 and is typical for a Galactic $\zeta$-type environment. The ionization balance as probed by the $5780 / 5797$ DIB ratio shows similar values $(\sim 6-8)$ for the LMC 30 Doradus and the UV exposed Galactic $\sigma$-environments. Lower values $(\sim 2)$ are found for the SMC wing (AzV 456) and the shielded Galactic $\zeta$-environment. For the SMC bar (i.e. $\mathrm{AzV} 18$ ) we find an intermediate value of $\sim 3$. Welty et al. (2006) found that the 5780, 5797 and $6284 \AA$ DIB strengths (per unit reddening) show no direct trends with the derived local ISRF.

The small particles that give rise to the observed increased attenuation of high energy photons (see Fig. 2) ensure that stable gas phase molecules are less susceptible to photoionisation as well as photodissociation. This suggests that even in such a harsh 
environment a small DIB carrier contribution is expected to survive and to be ionised. In low metallicity environments the decreased carbon abundance and thus complex carbonaceous molecule abundance would give rise to weaker absorption lines, although this is partly compensated by an increase in the ionisation fraction. Recent PDR models of diffuse clouds with SMC type extinction and metallicity suggest that for moderate ambient UV radiation fields a significant fraction of large carbonaceous molecules is ionised (Cox \& Spaans 2006). For stronger UV fields virtually all PAHs/fullerenes are ionised, including even those residing in the cores of diffuse clouds. In this instance, photodissociation processes are likely to become important and may, depending on the exact physical conditions of the cloud, result in a net destruction of small to intermediate size PAHs/fullerenes, i.e. DIB carriers. For the SMC-wing line-ofsight toward $\mathrm{AzV} 456$ calculations show that the relativele high dust-to-gas ratio (e.g. a higher metallicity and thus a higher expected carbon abundance) together with the less efficient attenuation of high energy (ionising) photons, could result in enhanced cation band strengths similar to those observed in Galactic linesof-sight (Cox \& Spaans 2006). Experimental studies and theoretical calculations show that larger PAHs ( $>50$ carbon atoms) are very photo-resistant (Omont 1986; Allain et al. 1996a,b; Le Page et al. 2001; Le Page et al. 2003) and so exist mostly in their cationic form in the diffuse SMC medium.

Grain disruption, either by a turbulent/disruptive medium or harsh UV radiation field, can be an additional important parameter in the formation or destruction of DIB carriers. From the $N(\mathrm{Na}$ I $) / N(\mathrm{Ca}$ II $)$ we conclude that the SMC bar and LMC 30 Doradus environments show also the highest level of turbulence, while the $\zeta$-type environment (HD 149757) is the most quiescent (Sect. 4.3.3; Table 9). The SMC wing and the Galactic $\sigma$-type cloud both have intermediate values. The much higher ratio in the Galaxy could be partly due to the higher Galactic dust content and thus easier depletion of calcium from the gas phase onto the grains. The SMC wing environment is more quiescent than in the bar. This is also indicated by the higher level of depletion of titanium from the gas phase.

\section{Summary and conclusion: DIBs, gas and dust in the SMC}

In this paper we outlined the properties of the gas and dust in the diffuse interstellar medium of the Small Magellanic Cloud (SMC) probed by seven different sightlines toward the early type stars AzV 456, AzV 18, AzV 398, AzV 214, AzV 65, Sk 191 and $\mathrm{AzV}$ 242. The observed lines-of-sight probe diffuse clouds in the SMC, with visual extinctions $A_{\mathrm{V}}$ not exceeding one magnitude (Table 6).

Diffuse interstellar bands are detected toward the star $\mathrm{AzV} 456$ which is located in the wing of the SMC (Ehrenfreund et al. 2002). We find upper limits for DIBs in the line-of-sight toward the other five stars, which are all located in the SMC bar region (Fig. 1). Given the $\mathrm{S} / \mathrm{N}$ and spectral resolution it would have been possible to (marginally) detect DIBs toward AzV 18, AzV 398 and Sk 191 of similar strength (per unit reddening) to those observed toward AzV 456. The non-detection of DIBs in these sightlines implies that the DIB carriers are relative lower in abundance as compared to the line-of-sight toward $\mathrm{AzV} 456$ (Table 2 and Fig. 4). Observed equivalent widths of the AzV 456 DIBs are similar, per unit reddening, to those observed toward Galactic $\zeta$ type targets (Table 9). The upper limits and measured values (Welty et al. 2006) for DIBs toward AzV 18 indicate reduced DIB strengths per unit reddening by at least a factor 2 to 5 . In any case, the SMC interstellar medium can sustain the chemistry that prevails in the Galactic ISM giving rise to the DIBs.

Properties of the dust and (atomic) gas in the lines-of-sight are derived. We computed the total-to-selective visual extinction ratio $R_{\mathrm{V}}$, visual extinction $A_{\mathrm{V}}$ and gas-to-dust ratio $N(\mathrm{HI}) / A_{\mathrm{V}}$ for the sightlines toward the observed SMC targets (Table 6). Column density profiles are constructed, and total integrated SMC column densties derived, for neutral ( $\mathrm{Na} \mathrm{I}, \mathrm{K} \mathrm{I}$ ) and ionised (Ti II, Ca II) atomic species. $\mathrm{CH}$ and $\mathrm{Ca}$ I transitions are observed in the line-of-sight toward AzV 456.

We infer a quiescent environment toward the wing target AzV 456 and Sk 191, while the SMC bar shows a more turbulent and UV exposed medium.

The sightlines toward the SMC bar stars lack the UV bump and show an excess of very small (silicate) particles and/or PAHs that absorb strongly in the far-UV above $6 \mu \mathrm{m}^{-1}$ (Fig. 2). It is seen that the sightlines with the weak/non-existent UV bump are also those that show no or very weak DIBs. Furthermore, it is striking that the one line-of-sight with a UV bump also shows prominent DIBs.

We identify two possible scenarios for the absence or depletion of DIB and bump carriers; 1) The difference in the shape of the extinction curve and the gas-to-dust ratio in the SMC bar region could be due to grain disruption/destruction, in particular of the carbonaceous $2175 \AA$ bump carrier material, by a strong and hard ambient UV field efficiently penetrating the diffuse gaseous medium (little dust shielding caused by higher gas-to-dust ratio) or by shocks in the diffuse medium, which leads also to an increased destruction rate for the DIB carriers; or 2) The harsh environment may prohibit the formation of bump material as well as DIB carriers, especially if these formation processes require grains, similar to the inferred lower $\mathrm{H}_{2}$-grain formation rate in low (molecular) density SMC sightlines (e.g. Lee et al. 2007).

Both scenarios depend on the lower metalicity and result in more gas and the formation of smaller units (grains or large molecules) that subsequently absorb and scatter photons of short wavelength, thus giving rise to a steep far-UV rise. In both cases the number of available complex carbonaceous material/molecules (per carbon and/or per hydrogen atom) is reduced.

For the SMC bar, the lower carbon abundance (i.e. inferred via the lower dust-to-gas ratio, the metallicity, the amount of cosmic carbon needed to reproduce the observed extinction curves) and the more efficient attenuation of ionising photons (reducing the ionisation rate) is expected to result in significantly weaker diffuse band strengths. However, this scenario does not entirely explain the observed weak 5780 and $5797 \AA$ DIBs toward AzV 18. This lower abundance of DIB carriers may be a result of enhanced efficiency of (photo)destruction processes.

More accurate determinations of the abundance, or the level of absence, of DIBs in the Small Magellanic Cloud interstellar medium are needed, as well as accurate modelling of cloud temperatures, densities and effective radiation fields, to get a more refined handle on the the effect of local environmental conditions and the formation and survival of the DIB carriers. Additional information on the UV extinction properties of the SMC dust, in particular the $2175 \AA$ bump and the far-UV rise, are required to understand the connection between their carriers and those of the DIBs.

In conclusion, our measurements indicate that the DIB characteristics depend on the local physical conditions and chemical composition of the interstellar medium of the SMC, which 
apparently determine the rate of formation (and/or) destruction of the DIB carriers. Understanding interstellar carbon chemistry is important to the study of dust formation, gas cooling, and extinction and shielding by dust. If the diffuse interstellar band carriers are carbonaceous molecules their identification would provide an important diagnostic probe of physical conditions and prevailing carbon compounds in the diffuse interstellar medium.

Acknowledgements. N.L.J.C. was supported by NOVA and P.E. by VI-NWO (016.023.003). We thank the referee for insightful comments. This research has made use of the SIMBAD database, operated at CDS, Strasbourg, France. This publication makes use of data products from the Two Micron All Sky Survey, which is a joint project of the University of Massachusetts and the Infrared Processing and Analysis Center/California Institute of Technology, funded by the National Aeronautics and Space Administration and the National Science Foundation. The Australia Telescope Compact Array and Parkes telescope are part of the Australia Telescope which is funded by the Commonwealth of Australia for operation as a National Facility managed by CSIRO. This research has made use of NASA's Astrophysics Data System.

\section{References}

Ádámkovics, M., Blake, G. A., \& McCall, B. J. 2005, ApJ, 625, 857

Adamson, A. J., Whittet, D. C. B., \& Duley, W. W. 1991, MNRAS, 252, 234

Allain, T., Leach, S., \& Sedlmayr, E. 1996a, A\&A, 305, 602

Allain, T., Leach, S., \& Sedlmayr, E. 1996b, A\&A, 305, 616

Asplund, M., Grevesse, N., \& Sauval, A. J. 2005, in Astronomical Society of the Pacific Conference Series, Vol. 336, Cosmic Abundances as Records of Stellar Evolution and Nucleosynthesis, ed. T. G. Barnes, III, \& F. N. Bash, 25 Azzopardi, M., \& Vigneau, J. 1977, A\&A, 56, 151

Azzopardi, M., \& Vigneau, J. 1982, A\&AS, 50, 291

Azzopardi, M., Lequeux, J., \& Rebeirot, E. 1988, A\&A, 202, L27

Bessell, M. S. 1991, A\&A, 242, L17

Bot, C., Boulanger, F., Lagache, G., Cambrésy, L., \& Egret, D. 2004, A\&A, 423, 567

Bothun, G. D. 1994, in Mass-Transfer Induced Activity in Galaxies, ed. I. Shlosman, 218

Bouchet, P., Lequeux, J., Maurice, E., Prevot, L., \& Prevot-Burnichon, M. L. 1985, A\&A, 149, 330

Bromage, G. E., \& Nandy, K. 1983, MNRAS, 204, 29

Browning, M. K., Tumlinson, J., \& Shull, J. M. 2003, ApJ, 582, 810

Cami, J., Sonnentrucker, P., Ehrenfreund, P., \& Foing, B. H. 1997, A\&A, 326, 822

Caplan, J., Ye, T., Deharveng, L., Turtle, A. J., \& Kennicutt, R. C. 1996, A\&A, 307,403

Cardelli, J. A., \& Savage, B. D. 1988, ApJ, 325, 864

Cardelli, J. A., Clayton, G. C., \& Mathis, J. S. 1988, ApJ, 329, L33

Cardelli, J. A., Clayton, G. C., \& Mathis, J. S. 1989, ApJ, 345, 245

Cartledge, S. I. B., Clayton, G. C., Gordon, K. D., et al. 2005, ApJ, 630, 355

Clayton, G. C., Gordon, K. D., \& Wolff, M. J. 2000, ApJS, 129, 147

Clayton, G. C., Wolff, M. J., Sofia, U. J., Gordon, K. D., \& Misselt, K. A. 2003, ApJ, 588, 871

Cox, A. N. 2000, Allen's astrophysical quantities (Allen's astrophysical quantities, 4th ed. (Publisher: New York: AIP Press; Springer), ed. Arthur N. Cox

Cox, N. L. J., \& Spaans, M. 2006, A\&A, 451, 973

Cox, N. L. J., Cordiner, M. A., Cami, J., et al. 2006, A\&A, 447, 991

Crawford, M. K., Tielens, A. G. G. M., \& Allamandola, L. J. 1985, ApJ, 293, L45

Cutri, R. M., Skrutskie, M. F., Van Dyk, S., et al. 2003, VizieR Online Data Catalog, 2246, 0

Danforth, C. W., Howk, J. C., Fullerton, A. W., Blair, W. P., \& Sembach, K. R. 2002, ApJS, 139, 81

Dartois, E., Muñoz Caro, G. M., Deboffle, D., \& d'Hendecourt, L. 2004, A\&A, 423, L33

Dartois, E., Muñoz Caro, G. M., Deboffle, D., Montagnac, G., \& D’Hendecourt, L. 2005, A\&A, 432, 895

Dekker, H., Nissen, P. E., Kaufer, A., et al. 2003, in Specialized Optical Developments in Astronomy, ed. E. Atad-Ettedgui, \& S. D’Odorico, Proc. SPIE, 4842, 139

D’Odorico, S., di Serego Alighieri, S., Pettini, M., et al. 1989, A\&A, 215, 21

Draine, B. T. 2003, ARA\&A, 41, 241
Drimmel, R., Cabrera-Lavers, A., \& López-Corredoira, M. 2003, A\&A, 409, 205 Dufour, R. J. 1984, in Structure and Evolution of the Magellanic Clouds, IAU Symp., 108, 353

Duley, W. W., \& Lazarev, S. 2004, ApJ, 612, L33

Ehrenfreund, P., Cami, J., Jiménez-Vicente, J., et al. 2002, ApJ, 576, L117

Evans, C. J., Crowther, P. A., Fullerton, A. W., \& Hillier, D. J. 2004a, ApJ, 610, 1021

Evans, C. J., Lennon, D. J., Trundle, C., Heap, S. R., \& Lindler, D. J. 2004b, ApJ, 607, 451

Evans, C. J., Lennon, D. J., Walborn, N. R., Trundle, C., \& Rix, S. A. 2004c, PASP, 116, 909

Falgarone, E., Pineau des Forets, G., \& Roueff, E. 1995, A\&A, 300, 870

Feast, M. W., Thackeray, A. D., \& Wesselink, A. J. 1960, MNRAS, 121, 337

Fitzpatrick, E. L. 1985, ApJS, 59, 77

Fitzpatrick, E. L. 1999, PASP, 111, 63

Foing, B. H., \& Ehrenfreund, P. 1994, Nature, 369, 296

Foing, B. H., \& Ehrenfreund, P. 1997, A\&A, 317, L59

Gordon, K. D., \& Clayton, G. C. 1998, ApJ, 500, 816

Gordon, K. D., Clayton, G. C., Misselt, K. A., Landolt, A. U., \& Wolff, M. J. 2003, ApJ, 594, 279

Heckman, T. M., \& Lehnert, M. D. 2000, ApJ, 537, 690

Herbig, G. H. 1995, ARA\&A, 33, 19

Hirashita, H. 1999, ApJ, 522, 220

Hobbs, L. M. 1974a, ApJ, 191, 381

Hobbs, L. M. 1974b, ApJ, 191, 395

Houziaux, L., Nandy, K., \& Morgan, D. H. 1985, MNRAS, 215, 5P

Hunter, I., Smoker, J. V., Keenan, F. P., et al. 2006, MNRAS, 367, 1478

Hutchings, J. B. 1966, MNRAS, 131, 299

Hutchings, J. B. 1980, PASP, 92, 592

Iglesias-Groth, S. 2004, ApJ, 608, L37

Issa, M. R., MacLaren, I., \& Wolfendale, A. W. 1990, A\&A, 236, 237

Jenkins, E. B. 1996, ApJ, 471, 292

Jenniskens, P., Ehrenfreund, P., \& Désert, F.-X. 1992, A\&A, 265, L1

Joseph, C. L., \& Jenkins, E. B. 1991, ApJ, 368, 201

Junkkarinen, V. T., Cohen, R. D., Beaver, E. A., et al. 2004, ApJ, 614, 658

Kim, S., Staveley-Smith, L., Dopita, M. A., et al. 2003, ApJS, 148, 473

Le Page, V., Snow, T. P., \& Bierbaum, V. M. 2001, ApJS, 132, 233

Le Page, V., Snow, T. P., \& Bierbaum, V. M. 2003, ApJ, 584, 316

Lee, D.-H., Pak, S., Van Dyke Dixon, W., \& van Dishoeck, E. F. 2007, ApJ, 655, 940

Leger, A., \& D'Hendecourt, L. 1985, A\&A, 146, 81

Leger, A., D'Hendecourt, L., Verstraete, L., \& Schmidt, W. 1988, A\&A, 203, 145

Lennon, D. J. 1997, A\&A, 317, 871

Lequeux, J. 1979, Rev. Mex. Astron. Astrofis., 4, 325

Massa, D., \& Fitzpatrick, E. 2003, in Astrophysics of Dust

Mathis, J. S. 1990, ARA\&A, 28, 37

McGee, R. X., \& Newton, L. M. 1986, PASA, 6, 471

McNamara, D. H., \& Feltz, K. A. 1980, PASP, 92, 587

Mennella, V., Colangeli, L., Bussoletti, E., Palumbo, P., \& Rotundi, A. 1998, ApJ, 507, L177

Misselt, K. A., Clayton, G. C., \& Gordon, K. D. 1999, ApJ, 515, 128

Morgan, D. H. 1987, QJRAS, 28, 328

Morton, D. C. 2003, ApJS, 149, 205

Nandy, K., Morgan, D. H., \& Houziaux, L. 1990, MNRAS, 245, 318

Omont, A. 1986, A\&A, 164, 159

Prevot, M. L., Lequeux, J., Prevot, L., Maurice, E., \& Rocca-Volmerange, B. 1984, A\&A, 132, 389

Rich, R. M. 1987, AJ, 94, 651

Rocca-Volmerange, B., Prevot, L., Prevot-Burnichon, M. L., Ferlet, R., \& Lequeux, J. 1981, A\&A, 99, L5

Routly, P. M., \& Spitzer, L. J. 1952, ApJ, 115, 227

Sanduleak, N. 1968, AJ, 73, 246

Sanduleak, N. 1969, AJ, 74, 877

Sarre, P. J. 2006, J. Mol. Spectros., 238, 1

Sauvage, M., \& Vigroux, L. 1991, in The Magellanic Clouds, IAU Symp., 148, 407

Savage, B. D., \& Sembach, K. R. 1991, ApJ, 379, 245

Schwering, P. B. W., \& Israel, F. P. 1989, A\&AS, 79, 79

Sembach, K. R., Danks, A. C., \& Savage, B. D. 1993, A\&AS, 100, 107

Siluk, R. S., \& Silk, J. 1974, ApJ, 192, 51

Snow, T. P., Welty, D. E., Thorburn, J., et al. 2002, ApJ, 573, 670

Sofia, U. J., Wolff, M. J., Rachford, B., et al. 2005, ApJ, 625, 167

Sofia, U. J., Gordon, K. D., Clayton, G. C., et al. 2006, ApJ, 636, 753

Sollerman, J., Cox, N., Mattila, S., et al. 2005, A\&A, 429, 559

Spaans, M. 1995, Ph.D. Thesis, University of Leiden, Leiden, The Netherlands Stanimirović, S., Staveley-Smith, L., Dickey, J. M., Sault, R. J., \& Snowden, S. L. 1999, MNRAS, 302, 417 
Stanimirović, S., Staveley-Smith, L., van der Hulst, J. M., et al. 2000, MNRAS, 315,791

Stanimirović, S., Staveley-Smith, L., \& Jones, P. A. 2004, ApJ, 604, 176

Staveley-Smith, L., Sault, R. J., Hatzidimitriou, D., Kesteven, M. J., \& McConnell, D. 1997, MNRAS, 289, 225

Staveley-Smith, L., Kim, S., Calabretta, M. R., Haynes, R. F., \& Kesteven, M. J. 2003, MNRAS, 339, 87

Thorburn, J. A., Hobbs, L. M., McCall, B. J., et al. 2003, ApJ, 584, 339

Tomita, S., Fujii, M., \& Hayashi, S. 2004, ApJ, 609, 220

Valencic, L. A., Clayton, G. C., Gordon, K. D., \& Smith, T. L. 2003, ApJ, 598, 369

Valencic, L. A., Clayton, G. C., \& Gordon, K. D. 2004, ApJ, 616, 912

Van der Zwet, G. P., \& Allamandola, L. J. 1985, A\&A, 146, 76

Vangioni-Flam, E., Lequeux, J., Maucherat-Joubert, M., \& Rocca-Volmerange, B. $1980, A \& A, 90,73$
Vladilo, G. 2002, ApJ, 569, 295

Vladilo, G., Crivellari, L., Molaro, P., \& Beckman, J. E. 1987, A\&A, 182, L59

Walborn, N. R. 1977, ApJ, 215, 53

Walraven, T., \& Walraven, J. H. 1977, A\&AS, 30, 245

Wayte, S. R. 1990, ApJ, 355, 473

Welty, D. E., Lauroesch, J. T., Blades, J. C., Hobbs, L. M., \& York, D. G. 2001, ApJ, 554, L75

Welty, D. E., Federman, S. R., Gredel, R., Thorburn, J. A., \& Lambert, D. L. 2006, ApJS, 165, 138

Winkler, H. 1997, MNRAS, 287, 481

York, B. A., Ellison, S. L., Lawton, B., et al. 2006, ApJ, 647, L29

Zhou, Z., Sfeir, M. Y., Zhang, L., et al. 2006, ApJ, 638, L105 


\section{Online Material}


N. L. J. Cox et al.: Interstellar gas, dust and diffuse bands in the SMC, Online Material $p 2$

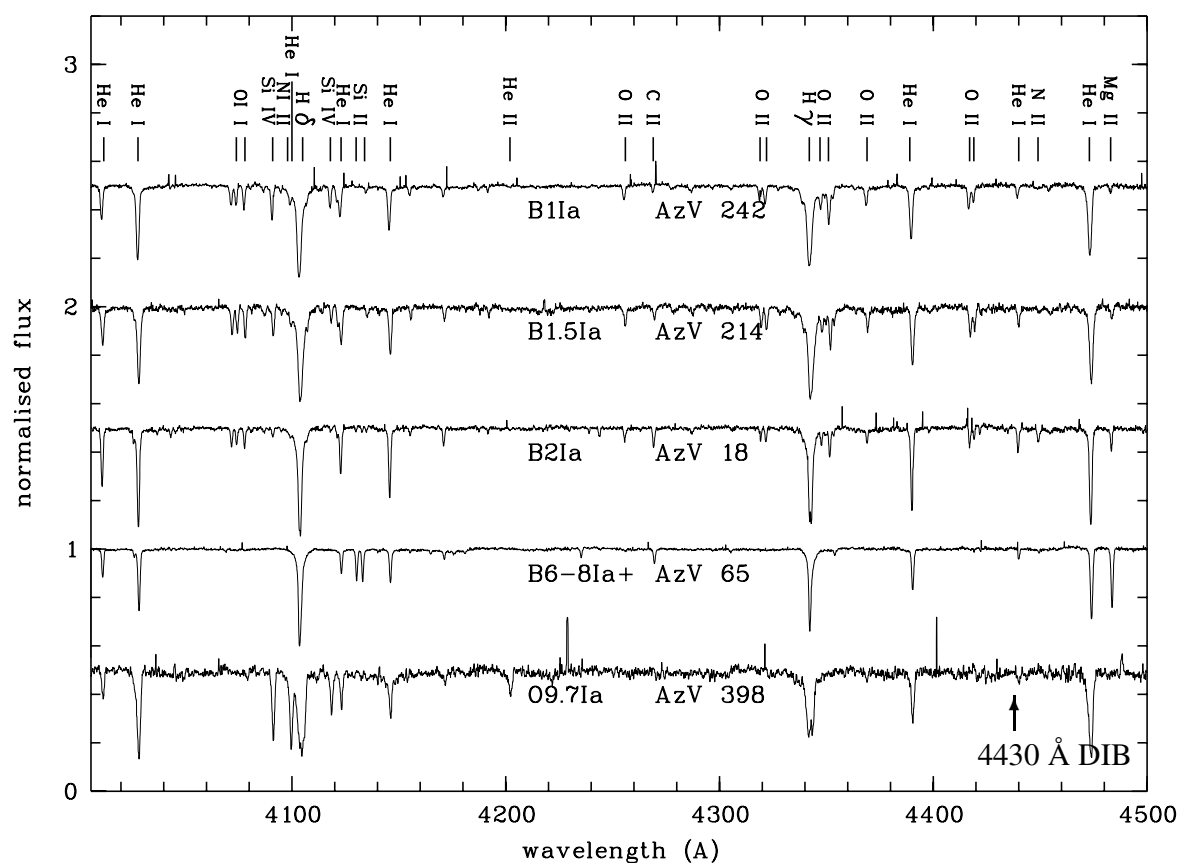

Fig. 8. The spectral region from 4005 to $4500 \AA$ of the targets obtained in August 2003. The identified stellar lines are indicated at the top of the figure. Due to the receding velocity of the SMC the stellar lines are shifted by about 2 to $2.5 \AA$. The spectra have been normalised to unity and displaced vertically for clarity. See the main text for a discussion on the derived/adopted spectral types of these targets. Note that their is no indication for the presence of the $4430 \AA$ DIB.

Table 7. $J, H$ and $K$ 2MASS photometry (Cutri et al. 2003), intrinsic colours (AN Cox [1] and Winkler [2]) and colour excesses for SMC targets $\mathrm{AzV} 65$ and $\mathrm{Sk}$ 191. $R_{\mathrm{V}}$ is calculated from the colour excesses using the relationships derived by Fitzpatrick (1999).

\begin{tabular}{|c|c|c|c|c|c|}
\hline Target & $\overline{B-V}$ & $\overline{V-J}$ & $\overline{V-H}$ & $\overline{V-K}$ & Source \\
\hline$\overline{A z V} 65$ & 0.13 & 0.43 & 0.522 & 0.561 & \\
\hline \multirow[t]{2}{*}{ B6Ia } & -0.02 & -0.09 & -0.05 & -0.07 & 1 \\
\hline & & -0.06 & -0.03 & -0.03 & 2 \\
\hline \multirow[t]{2}{*}{ B8Ia } & -0.06 & 0.02 & 0.09 & 0.07 & 1 \\
\hline & & 0.06 & 0.09 & 0.10 & 2 \\
\hline Sk 191 & -0.04 & -0.044 & -0.097 & -0.046 & \\
\hline \multirow[t]{2}{*}{ B1.5Ia } & -0.18 & -0.38 & -0.42 & -0.45 & 1 \\
\hline & & -0.325 & -0.355 & -0.39 & 2 \\
\hline Target & $E(B-V)$ & $E(V-J)$ & $E(V-H)$ & $E(V-K)$ & Source \\
\hline \multicolumn{6}{|l|}{$\mathrm{AzV} 65$} \\
\hline \multirow[t]{2}{*}{ B6Ia } & 0.15 & 0.52 & 0.572 & 0.631 & 1 \\
\hline & 0.15 & 0.49 & 0.552 & 0.591 & 2 \\
\hline \multirow[t]{2}{*}{$R_{\mathrm{V}}$} & & 4.8 & 4.6 & 4.7 & $4.6(0.25)$ \\
\hline & & 4.5 & 4.4 & 4.4 & $4.0(0.2 J)$ \\
\hline \multirow[t]{2}{*}{ B8Ia } & 0.19 & 0.41 & 0.432 & 0.491 & 1 \\
\hline & & 0.37 & 0.432 & 0.461 & 2 \\
\hline \multirow[t]{2}{*}{$R_{\mathrm{V}}$} & & 3.0 & 2.8 & 2.9 & \multirow{2}{*}{$2.75(0.15)$} \\
\hline & \multicolumn{4}{|c|}{ Sk 191} & \\
\hline \multirow[t]{2}{*}{ B1.5Ia } & 0.14 & 0.336 & 0.323 & 0.404 & 1 \\
\hline & & 0.281 & 0.258 & 0.344 & 2 \\
\hline \multirow[t]{2}{*}{$R_{\mathrm{V}}$} & & 3.3 & 2.8 & 3.3 & \multirow{2}{*}{$2.7(0.6)$} \\
\hline & & 2.8 & 2.2 & 2.8 & \\
\hline
\end{tabular}


N. L. J. Cox et al.: Interstellar gas, dust and diffuse bands in the SMC, Online Material $p 3$

Table 8. Galactic (foreground) atomic column densities derived via the construction of apparent column density velocity profiles and subsequent line profile integration. Error in $\log N$ is 0.02 dex for $\mathrm{Na}$ I, Ti II and Ca II K. Adopted line transition wavelengths and oscillator strengths from Morton (2003). Column densities toward Sk 191 and AzV 242 suffer significantly from line saturation. The smaller values for Ca II/Na I in the Galaxy are consistent with a higher UV in the SMC. Larger Galactic values for Ca II/Ti II by about 0.2 dex, indicate a higher sensitivity to the second ionisation potential in the SMC compared to the Galaxy.

\begin{tabular}{llllll}
\hline \hline \multicolumn{7}{c}{ Column density log $N\left(\mathrm{~cm}^{-2}\right)$} \\
& Na I D1/D2 & Ca II H/K & Ti II & Ca II/Na I & Ca II/Ti II \\
\hline AzV 456 & $11.97 / 11.86$ & $12.13 / 12.17$ & 11.98 & 0.24 & 0.17 \\
AzV 18 & $12.11 / 12.07$ & $12.11 / 12.09$ & 11.93 & 0.01 & 0.17 \\
AzV 214 & $12.35 / 12.23$ & $12.20 / 12.21$ & 12.13 & -0.09 & 0.07 \\
AzV 398 & $12.06 / 11.86$ & $12.29 / 12.30$ & 12.14 & 0.34 & 0.16 \\
AzV 65 & $12.32 / 12.14$ & $12.07 / 12.10$ & 11.91 & -0.24 & 0.17 \\
Sk 191 & $12.53 / 12.36$ & $12.14 / 12.20$ & 11.87 & -0.3 & 0.30 \\
AzV 242 & $12.16 / 12.02$ & $11.73 / 11.87$ & 12.04 & -0.29 & -0.24 \\
\hline
\end{tabular}

Table 9. Physical properties and conditions of the interstellar medium in the lines-of-sight toward the SMC targets AzV 456 and AzV 18 (this work), the LMC target Sk-69 223 (Cox et al. 2006) and the Galactic targets HD 62542, HD 144217 ( $\sigma$-type) and HD 149757 ( $\zeta$-type). UV lines are indicated by (uv) and the weak and strong doublet lines by (w) and (s), respectively.

\begin{tabular}{|c|c|c|c|c|c|c|c|}
\hline \multirow[b]{3}{*}{ Parameter } & \multirow[b]{3}{*}{ Unit } & \multicolumn{2}{|c|}{ SMC } & \multirow{3}{*}{$\begin{array}{c}\text { LMC } \\
\text { 30 Doradus } \\
\text { Sk-69 223 }\end{array}$} & \multicolumn{3}{|c|}{$\overline{\mathrm{MW}}$} \\
\hline & & Wing & Bar & & no bump & $\sigma$ & $\zeta$ \\
\hline & & $\mathrm{AzV} 456$ & AzV 18 & & HD 62542 & HD 144217 & HD 149757 \\
\hline$E_{B-V}$ & mag & 0.27 & 0.17 & 0.2 & 0.35 & 0.2 & 0.3 \\
\hline$R_{\mathrm{V}}$ & & 2.2 & 2.9 & 3.6 or 4.1 & 2.90 & 4.0 & 3.1 \\
\hline$A_{\mathrm{V}}$ & mag & 0.6 & 0.5 & $0.8(0.2)$ & 1.02 & 0.8 & 0.9 \\
\hline 5780 DIB & $\mathrm{m} \AA$ per $E_{B-V}$ & 256 & 135 & 725 & 151 & 800 & 220 \\
\hline 5797 DIB & $\mathrm{m} \AA$ per $E_{B-V}$ & 133 & 47 & 140 & 24 & 110 & 90 \\
\hline 6613 DIB & $\mathrm{m} \AA$ per $E_{B-V}$ & 181 & $<41$ & 100 & - & 200 & 143 \\
\hline $5780 / 5797$ & & 1.9 & 2.9 & 5.2 & 6.2 & 7.3 & 2.4 \\
\hline $\log N(\mathrm{Na} \mathrm{I})$ & $\mathrm{cm}^{-2}$ & 12.8 & 12.6 & 12.59 & - & 12.94 & 13.85 \\
\hline $\log N(\mathrm{~K} \mathrm{I})$ & $\mathrm{cm}^{-2}$ & $\begin{array}{r}13.6 \text { (uv) } \\
12.6 \mathrm{w} \text { (uv) } \\
12.7 \mathrm{~s} \text { (uv) }\end{array}$ & $\begin{array}{r}11.76 \mathrm{w} \\
11.36 \mathrm{~s}\end{array}$ & 11.03 & 11.90 & 11.19 & 11.85 \\
\hline $\log N(\mathrm{HI})$ & $\mathrm{cm}^{-2}$ & 21.3 & 21.95 & 21.46 & 20.93 & 21.10 & 20.73 \\
\hline $\log N(\mathrm{Ca}$ II $)$ & $\mathrm{cm}^{-2}$ & 12.3 & 13.1 & 12.68 & - & 11.73 & 11.76 \\
\hline$N(\mathrm{Na} \mathrm{I}) / N(\mathrm{Ca} \mathrm{II})$ & & 2.5 & 0.5 & 0.9 & - & 16.2 & 123 \\
\hline$N(\mathrm{HI}) / A_{\mathrm{V}}$ & $10^{21} \mathrm{~cm}^{-2} / \mathrm{mag}$ & 2.6 & 18.4 & 3.6 & 0.8 & 1.6 & 0.6 \\
\hline$f\left(H_{2}\right)$ & & 0.525 & 0.045 & $?$ & 0.603 & 0.05 & 0.32 \\
\hline $\log N\left(\mathrm{H}_{\mathrm{tot}}\right)$ & $\mathrm{cm}^{-2}$ & 21.75 & 21.95 & & & 21.13 & 21.15 \\
\hline$N\left(\mathrm{H}_{\mathrm{tot}}\right) / A_{\mathrm{V}}$ & $10^{21} \mathrm{~cm}^{-2} / \mathrm{mag}$ & 9.36 & 17.95 & & & 1.69 & 1.57 \\
\hline $5780 / Z$ & $\left(Z=A_{\mathrm{V}} / \mathrm{HI}\right)$ & 179 & $<442$ & 522 & 42 & 256 & 40 \\
\hline $5797 / Z$ & $\left(Z=A_{\mathrm{V}} / \mathrm{HI}\right)$ & 94 & $\leq 74$ & 101 & 7 & 35 & 16 \\
\hline
\end{tabular}

